\title{
Deep Learning for Diabetes: A Systematic Review
}

\author{
Taiyu Zhu, Student Member, IEEE, Kezhi Li, Member, IEEE, \\ Pau Herrero, Member, IEEE, and Pantelis Georgiou, Senior Member, IEEE
}

\begin{abstract}
Diabetes is a chronic metabolic disorder that affects an estimated 463 million people worldwide. Aiming to improve the treatment of people with diabetes, digital health has been widely adopted in recent years and generated a huge amount of data that could be used for further management of this chronic disease. Taking advantage of this, approaches that use artificial intelligence and specifically deep learning, an emerging type of machine learning, have been widely adopted with promising results. In this paper, we present a comprehensive review of the applications of deep learning within the field of diabetes. We conducted a systematic literature search and identified three main areas that use this approach: diagnosis of diabetes, glucose management, and diagnosis of diabetes-related complications. The search resulted in the selection of 40 original research articles, of which we have summarized the key information about the employed learning models, development process, main outcomes, and baseline methods for performance evaluation. Among the analyzed literature, it is to be noted that various deep learning techniques and frameworks have achieved stateof-the-art performance in many diabetes-related tasks by outperforming conventional machine learning approaches. Meanwhile, we identify some limitations in the current literature, such as a lack of data availability and model interpretability. The rapid developments in deep learning and the increase in available data offer the possibility to meet these challenges in the near future and allow the widespread deployment of this technology in clinical settings.
\end{abstract}

Index Terms-Diabetes, deep learning, deep neural networks, glucose management, diabetic complications, artificial intelligence

\section{INTRODUCTION}

Diabetes is a group of lifelong metabolic disorders caused by defective insulin secretion or impaired insulin action. The International Diabetes Federation estimates that there are 463 million people (95\% confidence interval: 369-601 million) living with diabetes in 2019, half of whom, however, remain undiagnosed, due to the complex pathogenesis of diabetes [1]. The global prevalence of diabetes is projected to significantly increase in the coming decade. Therefore, preventing and treating diabetes has been a heavy burden for national economies, healthcare systems, and personal medical expenditures, especially for low- and middle-income countries [2].

According to the etiopathology of diabetes, there are three main clinical categories: type 1 diabetes (T1D), type 2 diabetes (T2D), and gestational diabetes mellitus (GDM) [3]. Other categories due to specific causes include latent autoimmune diabetes of adulthood and maturity-onset diabetes of the

This work was supported by EPSRC EP/P00993X/1.

T. Zhu, P. Herrero, P. Georgiou are with Centre for Bioinspired Technology, Imperial College London, London, United Kingdom. (e-mail: \{taiyu.zhu17, pherrero, pantelis\}@imperial.ac.uk).

$\mathrm{K} . \mathrm{Li}$ is with Institute of Health Informatics, University College London, London, United Kingdom. (e-mail: ken.li@ucl.ac.uk). young. T1D occurs when the insulin-secreting $\beta$-cells of the pancreas are destroyed by the immune system [4]. People with T1D suffer from the absolute insufficiency of endocrine insulin produced by the pancreatic $\beta$-cell, hence they rely on exogenous delivery. T2D accounts for about $90 \%$ of people with diabetes, resulting from insulin resistance or insufficient insulin production. GDM appears during pregnancy and might require lifestyle interventions and exogenous insulin delivery to prevent complications in the infant. However, due to the increasing heterogeneity and lack of continuous monitoring, the early diagnosis and classification of diabetes are often difficult in practice [5].

The majority of people with diabetes needing exogenous insulin employ the so-called basal-bolus insulin therapy, which consists on measuring glucose levels with a glucose levels meter and delivering multiple daily injections (MDI) with an insulin pen or with an insulin pump (continuous subcutaneous insulin infusion (CSII)) [6].

For people living with diabetes, it is vital to maintain blood glucose (BG) levels in a normal range. Otherwise, hyperglycemia or hypoglycemia can cause short and long-term complications in microvascular and macrovascular, including neuropathy, nephropathy, retinopathy, stroke, cardiovascular disease, and peripheral vascular disease [7]. Nevertheless, BG control is challenging for people with diabetes, since there are plenty of daily factors that influence BG levels, such as meal ingestion, exercise, alcohol, illness, and stress. Thus selfmanagement, e.g., timely BG measurement, hormone delivery, and adherence to recommended lifestyle are quite important, but all of them require multidisciplinary knowledge in clinical practice, especially for children and adolescents [8]. Besides, due to the high inter and intra-population variability in the glucose kinetics process and pharmacokinetics, it is difficult to find an optimal therapeutic strategy for all people [9].

In recent decades, continuous glucose monitoring (CGM) systems [10]-[12] and closed-loop hormone delivery systems [13], [14], also known as the artificial pancreas (AP), have been widely researched, aiming at developing automatic glucose regulation and relieving the burden of glucose management. An AP system employs CGM, a closed-loop control algorithm, and an insulin pump to deliver insulin by CSII. It has been proven to effectively reduce glycaemic control and is recommended to some T1D cohorts [15]. Although the AP is currently the state of the art in insulin delivery, standard basal-bolus insulin therapy with a capillary blood glucose meter and MDI through an insulin pen remains a cost-effective treatment option, and in particular thanks to the recent improvements enabling wireless connectivity of these devices to a smartphone (i.e. smart pens and smart meters) have significantly enhanced this therapeutic option [16], [17]. 


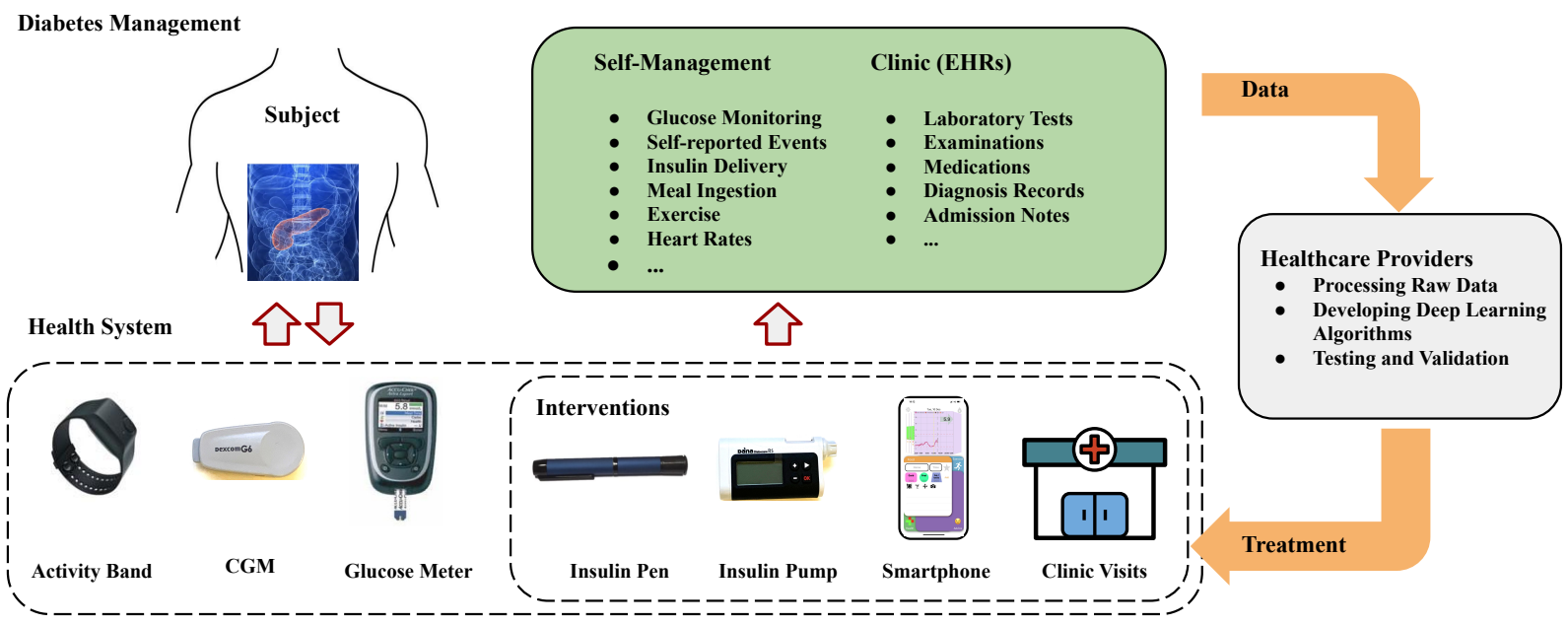

Fig. 1: The illustration of diabetes management, along with the large amount of data produced by self-management and clinical EHRs. The data is processed by healthcare providers to develop deep learning algorithms for the new treatments.

Furthermore, significant progress has been made in developing smartphone applications and the integration of portable devices, such as CGMs, for diabetes care [18] which allow users to $\log$ their daily events and ultimately use them to provide decision support. There is an increasing interest in integrating physical activity monitoring through exercise bands to enhance BG management [19]. Consequently, the wide use of wearable devices and digital systems in diabetes management and the increasing electronic health records (EHRs) in clinics have produced a large amount of available data, as depicted in Fig. 1. This current scenario offers tremendous opportunities to apply advanced methods of artificial intelligence (AI) in diabetes care to further improve the treatment of diabetes.

The medical datasets from multiple sources are often heterogeneous, high-dimensional, and sparse, and thus they are likely to be underused in clinical scenarios [20]. Fortunately, machine learning, as an increasingly successful AI branch, is powerful at discovering nonlinear correlations of high-dimensional data. The definition of machine learning is that systems are able to learn knowledge and patterns automatically from experience or existing data without being explicitly instructed [21]. Moreover, empowered by boosting computational capabilities, a frontier machine learning method, deep learning, has achieved recent success and improved performance surpassing state-ofart in many health domains [22]. Compared to conventional machine learning technology, deep learning allows the input of raw data and learns representation automatically by exploiting deep neural networks (DNNs), which require little feature engineering work on data pre-processing [23].

Although there have been comprehensive literature reviews on $\mathrm{AI}$ for diabetes [24]-[26], covering some conventional machine learning methods and statistical models, they still lack a systematic study focusing on deep learning applications for diabetes. As an emerging approach, deep learning has recently shown competitive performance in several important fields of diabetes, such as diabetic eye diseases [27]. Therefore, in this work, we specifically investigate the latest advances in deep learning technologies for diabetes care.

\section{DeEP Learning Overview}

Among the wide range of techniques and approaches in deep learning, we present the overview of several popular deep learning methods that are commonly applied to healthcare and, in particular, in the diabetes field. Deep learning originated from artificial neural networks (ANNs) inspired by the structure of biological neurons in the brain [28]. A standard ANN, as depicted in Fig. 2, comprises a number of nodes and three layers: input, hidden and output layer, to simulate the neuron behaviors by mathematical expressions. In general, an ANN gains the perceptions through an iterative training process called back-propagation but lacks generalization for supervised tasks [29]. By adding more hidden layers, deep learning extends the ANN structure to DNNs for better generalization, which extracts data features and learns representations with thousands or even millions of parameters [30]. The breakthroughs of computational hardware and software infrastructures largely accelerate the development of deep learning by increasing the size and depth of DNN models in the recent two decades [28]. Fig. 2 depicts five popular DNN architectures employed in diabetes research with the corresponding nodes, cells, and connections. Popular software frameworks to implement deep learning algorithms include Theano [31], Caffe [32], TensorFlow [33], CNTK [34], and PyTorch [35]. These frameworks support various programming languages and hardware acceleration, which help people efficiently build DNN models.

In general, most of the deep learning algorithms can be divided into supervised learning, unsupervised learning, and reinforcement learning. Classification and regression are common tasks in supervised learning, for which the labeled input data is used during iterative model optimization and backward propagation [29]. There are three supervised learning-based DNNs found in the literature of diabetes: deep multilayer perceptrons (DMLPs), convolutional neural networks (CNNs), and recurrent neural networks (RNNs). The DMLP, also known as a feed-forward neural network, uses the simple 
Deep Neural Networks (DNNs)

Artificial Neural Networks (ANNs)
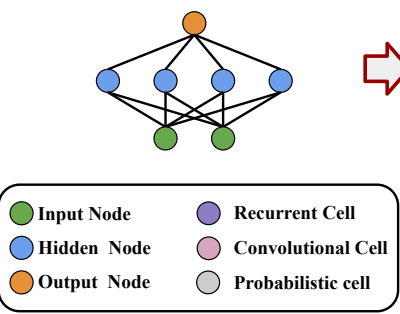

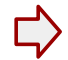

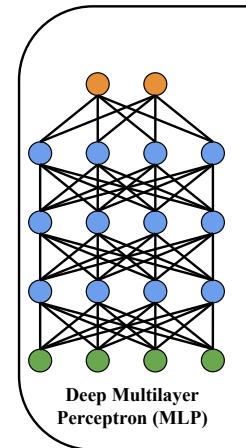

Supervised Learning

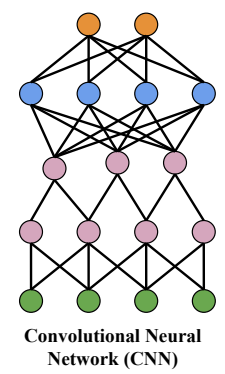

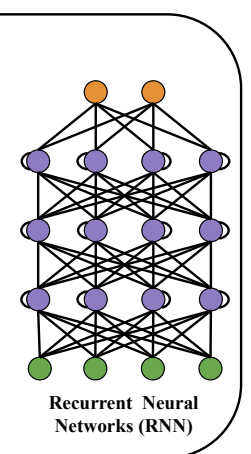

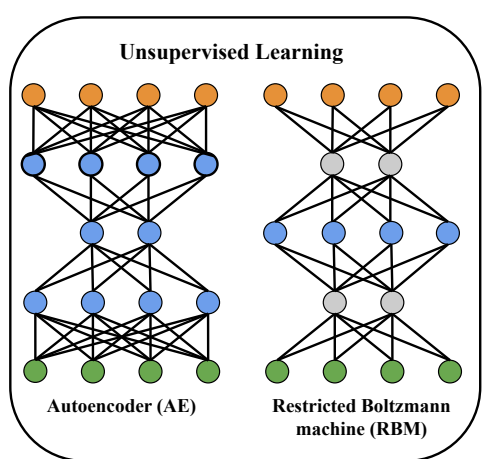

Fig. 2: The visualization of ANNs and DNNs. DNNs have an increasing number of hidden layers embedding the variants of neural nodes and cells. Higher-level feature maps are computed by the deep models. There are five popular DNN architectures for diabetes: DMLP, CNN, RNN, AE, and RBM.

connections between neurons, i.e. fully connected (FC) layers, and forms the basis of many DNN models. The term "deep" is highlighted to indicate the modes have deep architectures with more than three layers since multilayer perceptrons refer to both ANNs and DNNs in some studies. A DMLP is associated with a set of weight vectors, bias scalars, and the nonlinear activation functions, including sigmoid, tanh, and rectified linear units (ReLU) [28].

Leveraging convolutional layers as preceptors, CNNs can process the signals of multi-dimensional arrays and achieve superior performance on imaging tasks [36]. A sub-sampling layer, or pooling layer, is employed in most CNN architectures to aggregate feature maps. One major advantage of convolutional operations is to reduce the neuron connections between layers, as depicted in Fig. 2, which notably enhances the efficiency of model training through back-propagation. Empowered by the parallelized operations of graphics processing units (GPUs) and tensor processing units (TPUs) [37], various $\mathrm{CNN}$-based models have been applied to large-scale imaging recognition tasks, such as ImageNet database [38], and transformed to industry practices. In this regard, the popular CNN configurations found in literature are as follows: AlexNet [30], VGGNet [39], Inception (GoogLeNet) [40], and ResNet [41].

Different from other feed-forward neural networks, the input of an RNN contains the information at the previous timesteps. This feature makes RNNs powerful at processing sequential signals to capture temporal features. However, the difficulty of vanilla RNNs lies in the back-propagation training, where the gradient vanishing and exploding problems are likely to occur [42]. Fortunately, the advanced RNN cells, long short-term memory (LSTM) [43], and gated recurrent units (GRUs) [44], have overcome these problems by introducing gate functions and persevering long-term information. These RNN-based models have provided paradigms in numerous prediction and regression tasks, especially in natural language processing (NLP) and speech recognition. The latest trend in RNN is the attention mechanism [45], which allows models to focus on certain parts of input sequences and map the dependencies regardless of the distances.

Regarding unsupervised learning, the predefined labels or classes of inputs are not required for the model training. In this context, the algorithm aims at inferring the hidden structures and representations from the input datasets without supervision. Unsupervised learning is a powerful tool for data preprocessing, cluster analysis, density estimation, and dimension reduction. The autoencoder (AE) and the restricted Boltzmann machine (RBM) are the two basic architectures. The key feature of AEs is that its training target is the same as the input. Latent representations of the input are first transformed by an encoder, then fed to a decoder for reconstruction at the output. An RBM is another approach to map the representations by estimating probabilistic distribution over the input data, and thus it is also regarded as a generative model. Compared to standard Boltzmann machines, RBMs only allow the neuron connections that form a bipartite graph, to accelerate training processes. By stacking multiple RBMs, deep belief networks (DBNs) or deep Boltzmann machines can be constructed [46]. In most cases, DBNs are used as feature detectors to extract representations from data by unsupervised learning. However, supervised learning can be further performed to fine-tune the network weights and improve performance for certain learning tasks [47].

Deep reinforcement learning (DRL) surpass human professions in a variety of control problems with high-dimensional environments, where DNNs are employed as the approximators of policy, value-function, or system models. The network is trained by interacting with the environment consistently [48].

\section{Methodology}

Aiming at identifying and analyzing the benefits of deep learning within diabetes research, we conducted a systematic review by searching multiple public online databases, including PubMed, DBLP Computer Science Bibliography, and IEEEXplore. PubMed is a reputable database for biomedical and clinical research, while DBLP contains millions of 


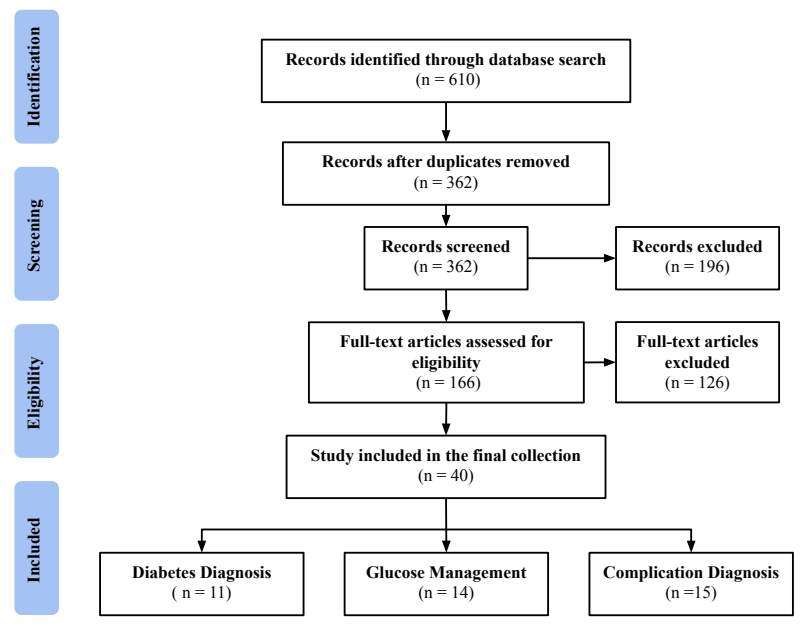

Fig. 3: PRISMA flow of selection process.

publications in the field of computer science. IEEE Xplore is a digital library that covers studies in engineering and allied fields. All three databases provide free and open-access search engines or interfaces without requiring institutional subscriptions like other databases (e.g. Ovid, Scopus, and Web of Science). Therefore, to facilitate the reproducibility of the search results, we have chosen open-access search engines. We restricted the search to English-language documents that were published between January 1, 2016 and March 31, 2020 (first quarter, Q1). The search was performed between April 5 and May 1, 2020, and was based on titles, abstracts, and metadata. We followed preferred reporting items for systematic review and meta-analyses (PRISMA) approach [49]. Fig. 3 summarizes the selection process.

\section{A. Search Strategies}

In our paper search, the keywords "diabetes", "glucose" and "artificial pancreas" were combined with the deep learning terms using Boolean operators AND/OR. The specific query searched was: ((diabetes OR glucose OR artificial pancreas) AND (deep learning OR deep neural network OR convolution neural network OR convolutional neural network OR recurrent neural network OR LSTM OR autoencoder OR boltzmann machine OR deep belief network)). After obtaining the results of an initial collection of relevant articles, we first excluded duplicated articles from different sources, then performed a manual inspection to evaluate the remaining based on inclusion criteria.

\section{B. Inclusion and Exclusion Criteria}

The studies included in this review are original and available full-text, focusing on deep learning applications in diabetes. The final collection of articles was organized into three categories based on the clinical application: diagnosis of diabetes, glucose management, and diagnosis of complications. Particularly, the included studies were expected to:

- present the details of datasets and data processing
- explicitly describe methods, e.g., the structure of DNNs

- evaluate model performance with standard metrics.

It should be noted that the application of diabetic retinopathy accounts for a large portion of the literature. Thus, in this area, we selected the works presenting DNN results of high originality, or large-scale clinical datasets. Abstracts, posters, technique reports, and reviews were excluded.

\section{Information Extraction}

From the selected collection of articles, we inspected the full-text and extracted key information to assess the deep learning applications. The following pre-defined categories were used to present the selected studies (Tables I, II] III).

1) Cases: We first summarized the specific application cases, i.e. scenarios, of the selected studies to identify the target of each work. For the studies with the available information of the types of diabetes, we have indicated them with $\dagger$ and $\ddagger$ for T1D and T2D, respectively.

2) Models: We present an overview of model architectures which includes a variety of DNN layers and the popular configurations, as mentioned in Section III. The details of hybrid structures and ensemble techniques are also included.

3) Data Sources: The source of input data is an essential factor for deep learning models. Many studies use more than one dataset, including public and private datasets, to validate the generalization of DNN models. Thus, this category summarizes the information regarding the employed datasets, e.g. sources, types, and formats. To facilitate future research to address the issues of data availability, we have highlighted the publicly available datasets with *

4) Development Process: This category summarizes the strategies for developing deep learning models, including preprocessing, training, validation, and testing. Although deep learning is good at extracting representations from raw data, these development steps need to be carefully designed, which impact on the functionality and reproducibility of the models.

5) Main Outcomes: The major outcomes with the corresponding metrics and criteria for performance evaluation, are included in this category. Some of the employed metrics in diabetes and complication diagnosis are sensitivity, specificity, and area under the curve (AUC); and root mean square error (RMSE) is common in glucose management. The results are consistent with the goals in the Cases category.

6) Baselines: In most selected studies, the authors implemented various baseline methods to compare with the performance of DNN algorithms. Many conventional statistic and machine learning methods are collected in this category, including logistic regression (LR), autoregression (AR), autoregressive integrated moving average (ARIMA), supporting vector machines (SVMs), random forests (RFs), naive Bayes (NB), k-nearest neighbors (KNN), latent variable model (LVX), principal component analysis (PCA), and decision trees (DTs). The best performance achieved by the baselines is also presented for the purpose of comparison using the metrics that are consistent with the Main Outcomes category. 


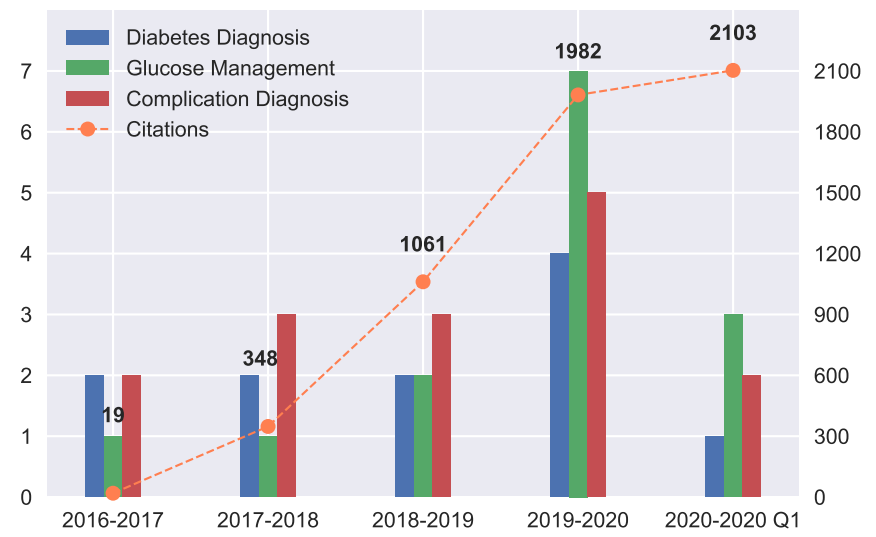

Fig. 4: Number of articles included in the collection grouped by the year of publication and application field. The orange dashed line indicates the number of citations in each year.

7) Limitations: As a review for an emerging methodology such as deep learning, this category collects the limitations that were identified for the selected studies, which could inspire future work and improve the learning performance in each application area.

\section{REsults}

The initial search yielded a total of 610 papers (PubMed (307), DBLP (31), and IEEE Xplore (272)), as shown in Fig. 3 After removing the duplicates, 362 papers remained. Then the papers were screened by the inclusion and exclusion criteria. We manually assessed the eligibility of the remaining papers by full-text inspection and included 40 papers in the final collection. Based on the application scenarios, we divided the final collection into three categories: diagnosis of diabetes $(\mathrm{n}=11)$, glucose management $(\mathrm{n}=14)$, and diagnosis of complications $(n=15)$. As shown in Fig. 4, most of the selected papers were published in recent two years, which indicates that the deep learning research for diabetes is a fairly new topic and its interest has been accelerating. In addition, we also calculated and plotted the number of citations of the selected papers in October 2020, according to Google Scholar. The details of the selected works are presented in chronological order in Tables IIII. III.

\section{A. Diagnosis of Diabetes}

The early diagnosis of diabetes can effectively improve the medical care and treatment for people living with diabetes. The standard diagnosis to confirm diabetes in clinics requires repeated glucose-based tests on hemoglobin A1c (HbA1c) and corresponding diagnostic criteria for different diabetes types [3]. However, due to the huge population and shortage of physicians in rural areas, the number of undiagnosed cases is significant and projected to increase in the future [61]. There is a high risk of developing diabetes without the onset of symptoms, especially for people with T2D, which could lead to long-term dysfunction of various organs and chronic complications [62].
Therefore, the need to detect onset diabetes or predict the diabetes risk arises, e.g. population screening and noninvasive systems. Table I presents the current efforts at developing deep learning decision-support algorithms for the diagnosis of diabetes. In particular, various supervised and unsupervised learning approaches strategies have been applied, where DMLP models are the most widely employed. The feed-forward structures and simple connections make DMLP a good option for a binary classifier on EHRs, while AEs and RBMs are used to extract underlying patterns of the data without supervision. It is noted that many studies have used the publicly available dataset called Pima Indian Diabetes (PID), from the University of California Irvine (UCI) repository [63]. It contains 768 instances with eight attributes and a binary label (diabetic or non-diabetic), which can be visualized by [64]. The Pima Indians have a higher prevalence of T2D than any population [65], making the PID dataset popular in machine learning research. An advantage of using this dataset with the same metrics is to easily compare the results with the previous work employing various machine learning methods. In Table [1 other public EHR datasets specific to the area of diagnosing diabetes include Mount Sinai Data Warehouse [66] and the Practice Fusion dataset [67]. Although these datasets are collected from different sources, they use common coding systems for diagnosis, namely the International Classification of Diseases ICD-9 and ICD-10. With these systems, researchers can easily locate the EHRs of patients with different types of diabetes and complications.

Nevertheless, the major limitation of applying deep learning on the PID dataset is the small number of patients and attributes. To prove the generalization of DNNs, the trained models need to be validated on a large population dataset. To this end, Miott et al. proposed a framework, namely Deep Patient, using a stack of denoising AEs to learn the representations from a large-scale dataset. The achieved AUC for the diagnosis of diabetes classification was 0.907 [50]. A recent study by Ryu et al. also employed a large dataset with 11,456 subjects [60]. They used a DMLP model to screen undiagnosed diabetes and achieved an AUC of $80.11 \%$ to detect undiagnosed diabetes. It is worth highlighting the data pre-processing step employed in these studies to extract related descriptors from the attributes of the patients, such as feature analysis and data normalization.

Moreover, the non-invasive detection of diabetes is emerging in several studies. Lekha et al. proposed a one-dimensional (1-D) CNN architecture to analyze the biomarkers in real-time breath signals for diabetes detection and classification [53]. The breath samples were collected by MOS sensors to analyze volatile organic compounds. The sensor array measured the content in a small gas chamber with an interval of 1000 seconds. Then the CNN classifier further processed these signals, which can reduce the need for feature selection and optimized the overall performance, compared to PCA, SVM, and singular value decomposition algorithm (SVD). In [54], the heart rate variability from electrocardiograms (ECG) was used as a marker to detect diabetes. The data was collected from a group of 40 people over a 10-minute duration. The ECG signals were sampled at $500 \mathrm{~Hz}$ with digital bandpass 
TABLE I: Summary of selected articles from the literature on diabetes diagnosis.

\begin{tabular}{|c|c|c|c|c|c|c|}
\hline Ref. & Cases & Models & Data Sources & Development Process & Main Outcomes & Baselines \\
\hline [50] & $\begin{array}{l}\text { Classification } \\
\text { of diabetes }\end{array}$ & Denosing AE & $\begin{array}{l}\text { Mount Sinai Data } \\
\text { Warehouse }^{\star} \text { (ICD-9) }\end{array}$ & $\begin{array}{l}\text { Normalization; pre-process to } \\
\text { obtain raw features; the data of } \\
\text { training, validation and testing: } \\
704,587,5000,76,214 \text { patients }\end{array}$ & AUC: 0.907 & $\begin{array}{l}\text { Original } \\
\text { descriptors, PCA } \\
(0.861)\end{array}$ \\
\hline [51] & $\begin{array}{l}\text { Prediction } \\
\text { of diabetes } \ddagger\end{array}$ & $\begin{array}{l}\text { Modified } \\
\text { LSTM, } \\
\text { attention } \\
\text { pooling layer }\end{array}$ & $\begin{array}{l}\text { An EHR dataset from } \\
\text { a regional hospital } \\
\text { ( } 7191 \text { patients, } \\
\text { ICD-10) }\end{array}$ & $\begin{array}{l}\text { The split for training, validation } \\
\text { and testing: } 2 / 3,1 / 6 \text { and } 1 / 6 \text { from } \\
53,208 \text { admissions }\end{array}$ & $\begin{array}{l}\text { Precision of diagnosis, } \\
\text { intervention, unplanned } \\
\text { readmission: } 66.2 \% \text {, } \\
78.7 \%, 79.0 \%\end{array}$ & $\begin{array}{l}\text { SVM, RF, plain } \\
\text { RNN, LSTM } \\
(65.7 \%, 78.2 \% \text {, } \\
75.9 \%)\end{array}$ \\
\hline [52] & $\begin{array}{l}\text { Detection of } \\
\text { diabetes }\end{array}$ & $\begin{array}{l}\text { RBM and } \\
\text { RNN }\end{array}$ & $\begin{array}{l}\text { PID dataset from } \\
\text { UCI repository }\end{array}$ & $\begin{array}{l}\text { Feature selection by RFs; min-max } \\
\text { normalization; the ratio for training } \\
\text { and testing data: } 80 \%, 20 \%\end{array}$ & $\begin{array}{l}\text { Sensitivity and } \\
\text { precision: } 90.66 \% \text {, } \\
75 \%\end{array}$ & N/A \\
\hline |53 & $\begin{array}{l}\text { Prediction } \\
\text { of } \\
\text { diabetes }^{\dagger \ddagger}\end{array}$ & $\begin{array}{l}\text { Modified 1-D } \\
\text { CNN and FC } \\
\text { layers }\end{array}$ & $\begin{array}{l}25 \text { breath samples } \\
\text { collected by MOS } \\
\text { sensors with } 1000-\mathrm{sec} \\
\text { intervals }\end{array}$ & $\begin{array}{l}\text { The data for training and testing: } \\
15 \text { samples, } 10 \text { samples; leave-one } \\
\text { out cross-validation }\end{array}$ & $\begin{array}{l}\text { AUC of T1D, T2D, } \\
\text { healthy subjects: } \\
0.9659,0.9625,0.9644\end{array}$ & SVD, SVM, PCA \\
\hline [54 & $\begin{array}{l}\text { Detection of } \\
\text { diabetes }\end{array}$ & $\begin{array}{l}\text { 5-layer CNN, } \\
\text { LSTM, and } \\
\text { SVM }\end{array}$ & $\begin{array}{l}\text { ECG data sampled at } \\
500 \mathrm{~Hz} \text { with digital } \\
\text { bandpass filtering and } \\
\text { thresholding collected } \\
\text { from } 40 \text { people }\end{array}$ & $\begin{array}{l}\text { Heart rate variability (HRV) data } \\
\text { from } 71 \text { ECG datasets (each } \\
\text { contains } 1000 \text { samples); } 5 \text { fold } \\
\text { cross-validation }\end{array}$ & $\begin{array}{l}\text { Validation accuracy: } \\
95.7 \%\end{array}$ & $\begin{array}{l}\text { Previous work } \\
\text { using HRV }\end{array}$ \\
\hline [55] & $\begin{array}{l}\text { Detection of } \\
\text { diabetes } \\
\end{array}$ & $\begin{array}{l}\text { DMLP with } \\
\text { dropout }\end{array}$ & $\begin{array}{l}\text { PID dataset from } \\
\text { UCI repository }\end{array}$ & $\begin{array}{l}\text { The ratio of training and validation } \\
\text { data: } 90 \% \text { and } 10 \%\end{array}$ & Accuracy: $88.41 \%$ & $\begin{array}{l}\text { Previous work on } \\
\text { the same dataset }\end{array}$ \\
\hline [56 & $\begin{array}{l}\text { Prediction } \\
\text { of diabetes } \ddagger\end{array}$ & DMLP & $\begin{array}{l}\text { A population dataset } \\
\text { ( } 4814 \text { participants,the } \\
\text { majority are } \\
\text { overweight) }\end{array}$ & $\begin{array}{l}\text { Data cleaning (imputing missing } \\
\text { values with the median); the ratio } \\
\text { of training and testing data: } 80 \% \\
\text { and } 20 \% \text { from } 656 \text { T2D subjects }\end{array}$ & $\begin{array}{l}\text { AUC without and with } \\
\text { HbA1c: } 0.703,0.840\end{array}$ & $\begin{array}{l}\text { SVM } \\
(0.679,0.825)\end{array}$ \\
\hline |57| & $\begin{array}{l}\text { Prediction } \\
\text { of the onset } \\
\text { T2 } \mathrm{D}^{\ddagger}\end{array}$ & $\begin{array}{l}\text { DMLP and a } \\
\text { linear model }\end{array}$ & $\begin{array}{l}\text { Practice Fusion } \\
\text { dataset }(9948 \\
\text { patients, ICD-9) }\end{array}$ & $\begin{array}{l}\text { Feature extraction by grouping } \\
1312 \text { features; the ratio of training } \\
\text { and validation data: } 70 \% \text {, testing } \\
\text { data: } 30 \% ; 10 \text {-fold cross-validation }\end{array}$ & $\begin{array}{l}\text { Sensitivity: } 31.17 \% \text {, } \\
\text { AUC: } 84.13 \%\end{array}$ & $\begin{array}{l}\text { RF }(29.12 \% \\
16.07 \%)\end{array}$ \\
\hline [58] & $\begin{array}{l}\text { Detection of } \\
\text { diabetes } \ddagger\end{array}$ & $\begin{array}{l}2 \text { layer } \mathrm{AE} \\
\text { and a softmax } \\
\text { layer }\end{array}$ & $\begin{array}{l}\text { PID dataset from } \\
\text { UCI repository }\end{array}$ & $\begin{array}{l}\text { Training the layer one by one with } \\
\text { previous output; fine-tuning by } \\
\text { supervised learning }\end{array}$ & $\begin{array}{l}\text { Sensitivity: } 87.92 \% \text {, } \\
\text { specificity: } 83.41 \% \text {, } \\
\text { accuracy: } 86.26 \%\end{array}$ & $\begin{array}{l}\text { Previous work on } \\
\text { the same dataset }\end{array}$ \\
\hline [59] & $\begin{array}{l}\text { Prediction } \\
\text { of diabetes } \ddagger\end{array}$ & DBN & $\begin{array}{l}\text { PID dataset from } \\
\text { UCI repository }\end{array}$ & $\begin{array}{l}\text { Min-max normalization; feature } \\
\text { selection by PCA; pre-training for } \\
\text { RBMs; supervised fine-tuning }\end{array}$ & $\begin{array}{l}\text { Sensitivity: } 100 \%, \mathrm{~F} 1 \\
\text { score: } 0.808\end{array}$ & $\begin{array}{l}\text { DT, LR, RF, } \\
\text { SVM, NB }(75.9 \% \text {, } \\
0.760)\end{array}$ \\
\hline 60 & $\begin{array}{l}\text { Detection of } \\
\text { undiagnosed } \\
\text { diabetes }^{\ddagger}\end{array}$ & $\begin{array}{l}2 \text { hidden } \\
\text { layer DMLP } \\
\text { with dropout }\end{array}$ & $\begin{array}{l}\text { An EHR dataset from } \\
\text { a national survey } \\
(31,098 \text { subjects, } 4 \\
\text { years })\end{array}$ & $\begin{array}{l}\text { Combining } 2013-2016 \\
\text { datasets;selecting features by LR; } \\
\text { the data of training and testing: } \\
11456 \text { and } 4444 \text { subjects }\end{array}$ & AUC: $80.11 \%$ & $\begin{array}{l}\text { LR, KNN, SVM, } \\
\text { AdaBoost, } \\
\text { Gaussian NB, RF } \\
(79.05 \%)\end{array}$ \\
\hline
\end{tabular}

filtering and thresholding operations to reduce noise during the real-time detection. After deriving the heart rate time with the Pan-Tompkins algorithm, the study developed a hybrid deep learning model with CNN, LSTM, and SVM, and achieved a validation accuracy of $95.7 \%$.

\section{B. Glucose Management}

The goal of glucose management in diabetes is to keep BG levels in the euglycemia region and avoid undesired glycemic events (i.e. hypoglycemia and hyperglycemia). Taking advantage of the digitization of diabetes self-management (Fig. 1), the development of deep learning has been significantly accelerated (Fig. 4). There are several sub-domains in glucose management which can be differentiated: BG level prediction, BG anomalies detection, insulin delivery control, and daily-life decision support.

Among these, BG level prediction has attracted increasing attention in recent years. An accurate BG prediction enables early interventions to prevent BG anomalies (i.e. hypoglycemia and hyperglycemia) and assists sensor-augmented insulin pumps (e.g. predictive low-glucose insulin suspend) and AP systems (e.g. model predictive control) to deliver optimal insulin and/or glucagon doses. The use of smartphone applications allows people to report the exogenous events that influence BG levels. By temporally aligning CGM measurements with these self-reported events, such as meal composition and insulin dosage, a multivariate time series can be formed and processed by deep learning models. Normally, the prediction horizon $(\mathrm{PH})$ for short and long-term forecasting is 30 minutes and greater than 60 minutes respectively. In this scenario, the RNN-based architecture is a powerful tool, referring to its success in temporal sequence processing and regression. Augmented by LSTM cells, the RNN is the most widely used method for glucose prediction in Table II Mirshekarian et al. proposed an LSTM model for 30 and 60minute prediction, which outperforms the engineered physiological model (EPM) with SVR [69]. EPM is a continuous dynamic model used to calculate the system states, which comprises the compartments of meal absorption dynamics, insulin absorption dynamics, and glucose-insulin dynamics. They further introduced a neural attention layer to emulate the case-based prediction by a memory module [76]. Besides, 
TABLE II: Summary of selected articles from the literature on glucose management.

\begin{tabular}{|c|c|c|c|c|c|c|}
\hline Ref. & Cases & Models & Data Sources & Development Process & Main Outcomes & Baselines \\
\hline 68 & $\begin{array}{l}\text { Detection of } \\
\text { hypoglycemia }^{\dagger}\end{array}$ & $\begin{array}{l}\text { DBN by } \\
\text { stacking RBMs }\end{array}$ & $\begin{array}{l}15 \text { T1D children } \\
\text { monitored for } \\
\text { 10-hour overnight }\end{array}$ & $\begin{array}{l}\text { Calculating QT correlation and heart } \\
\text { rate; training and testing data: } 10 \text { and } \\
5 \text { subjects }\end{array}$ & $\begin{array}{l}\text { Sensitivity: } 79.70 \% \text {, } \\
\text { specificity: } 50.00 \%\end{array}$ & $\begin{array}{l}\text { DBN based on } \\
\text { ANNs }(76.28 \% \text {, } \\
50.40 \%) \text {, multiple } \\
\text { regression }\end{array}$ \\
\hline |69 & $\begin{array}{l}\text { Prediction } \\
\text { of } \mathrm{BG} \\
\text { levels }^{\dagger}\end{array}$ & $\begin{array}{l}\text { LSTM and a } \\
\text { linear layer }\end{array}$ & $\begin{array}{l}\text { A clinical database } \\
\text { with the T1D data } \\
\text { over } 1600 \text { days }\end{array}$ & $\begin{array}{l}\text { Pre-training; linear interpolation; } \\
\text { training and testing data: } 5 \text { patients in } \\
400 \text { days, another } 5 \text { patients with } 200 \\
\text { samples }\end{array}$ & $\begin{array}{l}\text { RMSE for } 30,60-\mathrm{min} \\
\text { PH: } 21.4,38.0 \mathrm{mg} / \mathrm{dL}\end{array}$ & $\begin{array}{l}\text { ARIMA, EPM } \\
\text { with SVR }(21.6 \text {, } \\
39.2 \mathrm{mg} / \mathrm{dL})\end{array}$ \\
\hline [70] & $\begin{array}{l}\text { Prediction } \\
\text { of BG } \\
\text { levels }^{\dagger}\end{array}$ & $\begin{array}{l}\text { LSTM, } \\
\text { Bidirectional } \\
\text { LSTM and } 3 \\
\text { FC layers }\end{array}$ & $\begin{array}{l}\text { (1) GoCarb dataset } \\
\text { (20 adults), (2) } \\
\text { UVa/Padova T1D } \\
\text { simulator (11 } \\
\text { adults) }\end{array}$ & $\begin{array}{l}\text { Pre-training on (2); linear interpolation } \\
\text { on (1); cross-validation }(67 \%, 33 \%) \text {; } \\
\text { testing data: } 26 \text { sub-dataset from (1), } \\
1791 \pm 141 \text { CGM samples }\end{array}$ & $\begin{array}{l}\text { RMSE for } 30,45, \\
60-\mathrm{min} \mathrm{PH}: 11.63 \text {, } \\
21.75,36.92 \mathrm{mg} / \mathrm{dL}\end{array}$ & $\begin{array}{l}\text { ARIMA, SVR } \\
(11.69,22.14 \\
37.42 \mathrm{mg} / \mathrm{dL})\end{array}$ \\
\hline [71 & $\begin{array}{l}\text { Prediction } \\
\text { of BG } \\
\text { levels }\end{array}$ & $\begin{array}{l}\text { Deep } \\
\text { sequential } \\
\text { polynomial } \\
\text { multi-output } \\
\text { model (RNN) }\end{array}$ & $\begin{array}{l}40 \text { T1D subjects } \\
\text { over } 1900 \text { days }\end{array}$ & $\begin{array}{l}\text { The ratio of training,validation and } \\
\text { testing data: } 85 \%, 7.5 \% \text { and } 7.5 \% \\
\text { from } 555,000 \text { CGM samples }\end{array}$ & $\begin{array}{l}\text { Absolute percentage } \\
\text { error for } 30 \text {-min PH: } \\
4.87\end{array}$ & $\begin{array}{l}\text { Linear } \\
\text { extrapolation, RF, } \\
\text { RNN(LSTM, 5.3) }\end{array}$ \\
\hline |72| & $\begin{array}{l}\text { Glycemic } \\
\text { control }^{\dagger}\end{array}$ & $\begin{array}{l}\text { CNN } \\
\text { (Inception-v3) }\end{array}$ & $\begin{array}{l}\text { Food- } 101 \text { dataset } \\
(101 \text { classes, } \\
101,000 \text { images })^{\star}\end{array}$ & $\begin{array}{l}\text { Image augmentation; pre-training on } \\
\text { ImageNet dataset; training and testing } \\
\text { data: } 75,750,25,250 \text { images }\end{array}$ & $\begin{array}{l}\text { Time in rage (TIR) of } \\
70-180 \mathrm{mg} / \mathrm{dL}: \\
91.76 \%, \text { top-1 } \\
\text { accuracy of the image } \\
\text { classification: } 81.65 \%\end{array}$ & $\begin{array}{l}\text { Standard } \\
\text { controller in } \\
\text { UVa/Padova T1D } \\
\text { simulator (TIR: } \\
78.8 \% \text { ) }\end{array}$ \\
\hline |73 & $\begin{array}{l}\text { Prediction } \\
\text { of BG } \\
\text { levels }{ }^{\ddagger}\end{array}$ & $\begin{array}{l}\text { LSTM with } \\
\text { dynamic time } \\
\text { warping }\end{array}$ & $\begin{array}{l}\text { A dataset from a } \\
\text { randomized trial }(26 \\
\text { adults, smartphone } \\
\text { group }(\mathrm{n}=11))\end{array}$ & $\begin{array}{l}\text { Pre-processing; transfer learning; } \\
\text { min-max normalization across patients; } \\
\text { training, validation and testing data: } \\
\text { CGM samples of } 120,30 \text { and } 30 \text { days }\end{array}$ & $\begin{array}{l}\text { Clark Error Grid } \\
\text { zones of next-day PH } \\
\text { (A: } 84.12 \text {, B: } 15.16 \\
\text { C: } 0, \text { D: } 0.72, \text { E: } 0) \%\end{array}$ & $\begin{array}{l}\text { ANN, KNN, ridge } \\
\text { regression (A: } \\
83.03 \%) \text {, kernel } \\
\text { ridge regression, } \\
\text { moving average }\end{array}$ \\
\hline |74| & $\begin{array}{l}\text { Prediction } \\
\text { of BG } \\
\text { levels }\end{array}$ & $\begin{array}{l}\text { CNN, LSTM } \\
\text { and } 2 \text { FC } \\
\text { layers }\end{array}$ & $\begin{array}{l}\text { (1)UVa/Padova T1D } \\
\text { simulator }(10 \\
\text { adults), (2) } 10 \\
\text { clinical subjects }\end{array}$ & $\begin{array}{l}\text { Using Gaussian filter to remove } \\
\text { outliers; training and testing data: } 50 \% \\
\text { and 50\% }\end{array}$ & $\begin{array}{l}\text { RMSE for 30, 60-min } \\
\text { PH: } 9.38,18.87(1) \\
21.07,33.27(2) \\
\mathrm{mg} / \mathrm{dL}\end{array}$ & $\begin{array}{l}\text { SVR }(22.00,34.35 \\
\text { (2)), LVX, neural } \\
\text { network, AR }\end{array}$ \\
\hline |75 & $\begin{array}{l}\text { Prediction } \\
\text { of } \mathrm{BG} \\
\text { levels }^{\dagger}\end{array}$ & $\begin{array}{l}\text { LSTM and a } \\
\text { FC layer }\end{array}$ & $\begin{array}{l}\text { RT_CGM dataset } \\
\text { (the population of } \\
451 \text { patients) }\end{array}$ & $\begin{array}{l}\text { Removing sequences with low quality; } \\
\text { Tikhonov regularization; training and } \\
\text { testing data: } 304,450 \text { and } 94128 \\
\text { samples }\end{array}$ & $\begin{array}{l}\text { RMSE for } 30,45, \\
60-\min \mathrm{PH}: 19.47 \text {, } \\
26.47,32.38 \mathrm{mg} / \mathrm{dL}\end{array}$ & $\begin{array}{l}\text { AR, ANN, } \\
\text { standard RNN, } \\
\text { non-linear AR } \\
(24.66,32.33 \\
38.58 \mathrm{mg} / \mathrm{dL})\end{array}$ \\
\hline |76 & $\begin{array}{l}\text { Prediction } \\
\text { of BG } \\
\text { levels }\end{array}$ & $\begin{array}{l}\text { Memory- } \\
\text { Augmented } \\
\text { LSTM with } \\
\text { neural attention } \\
\text { weights }\end{array}$ & $\begin{array}{l}\text { (1) OhioT1DM } \\
\text { dataset }^{\star},(2) \text { AIDA } \\
\text { Simulator, } \\
\text { (3)UVa/Padova T1D } \\
\text { simulator }\end{array}$ & $\begin{array}{l}\text { Pre-training; linear interpolation and } \\
\text { extrapolation; testing, validation and } \\
\text { training data: last } 10 \text { days, previous } 10 \\
\text { days, rest days (1); } 400,100 \text {, and } 100 \\
\text { days (2); } 70,10 \text { and } 10 \text { days (3) }\end{array}$ & $\begin{array}{l}\text { RMSE for } 30,60-\text { min } \\
\text { PH: } 18.74,30.63(1) \text {; } \\
1.23,2.27(2) ; 2.93 \text {, } \\
4.92(3) \text { with input of } \\
\text { CGM, insulin and } \\
\text { meal events }\end{array}$ & $\begin{array}{l}\text { ARIMA ( } 20.17, \\
33.47(1) ; 5.59 \\
16.48(2) ; 12.00 \\
18.66(3))\end{array}$ \\
\hline |77| & $\begin{array}{l}\text { Prediction } \\
\text { of BG } \\
\text { levels }^{\dagger}\end{array}$ & $\begin{array}{l}\text { Dilated CNN } \\
\text { (residual and } \\
\text { parameterized } \\
\text { skip } \\
\text { connections) }\end{array}$ & $\begin{array}{l}\text { (1) UVa/Padova } \\
\text { T1D simulator, (2) } \\
\text { ABC4D dataset, (3) } \\
\text { OhioT1DM dataset }^{\star}\end{array}$ & $\begin{array}{l}\text { Ruling out outliers; } \\
\text { interpolation/extrapolation; label } \\
\text { transformation; training, validation and } \\
\text { testing set: } 45 \%, 5 \%, 50 \%(1,2) ; 40 \\
\text { and days (3) }\end{array}$ & $\begin{array}{l}\text { RMSE for 30, 60-min } \\
\text { PH: 8.88, } 19.90(1) \text {; } \\
\text { 19.19, 31.78 (2); } \\
19.28,31.83(3)\end{array}$ & $\begin{array}{l}\text { SVR }(21.75,34.31 \\
(3)), \text { LVX (12.25, } \\
22.41 \text { (1)), neural } \\
\text { network (20.42, } \\
33.13 \text { (2)), AR }\end{array}$ \\
\hline [78] & $\begin{array}{l}\text { Glycemic } \\
\text { control }^{\dagger}\end{array}$ & $\begin{array}{l}\text { Deep } \\
\text { Q-network with } \\
\text { GRU or 1-D } \\
\text { CNN }\end{array}$ & $\begin{array}{l}\text { UVA/Padova } \\
\text { simulator ( } 30 \text { virtual } \\
\text { subjects) }\end{array}$ & $\begin{array}{l}\text { Using CGM and insulin data in past } \\
24 \text { hours as the states, action setting: } \\
\{0 \text {, basal rate, } 5 * \text { basal rate }\} \text {; testing in } \\
10 \text { days }\end{array}$ & $\begin{array}{l}\text { Average risk index } \\
\text { for the virtual } \\
\text { subject: } 9.26\end{array}$ & $\begin{array}{l}\text { Proportional- } \\
\text { integral-derivative } \\
\text { control }(11.80)\end{array}$ \\
\hline [79 & $\begin{array}{l}\text { Prediction } \\
\text { of BG } \\
\text { levels }\end{array}$ & $\begin{array}{l}2 \text { branches of } \\
\text { LSTM cells } \\
\text { (past and future } \\
\text { information) }\end{array}$ & $\begin{array}{l}\text { (1) UVa/Padova } \\
\text { T1D simulator (100 } \\
\text { adults), (2) Padova } \\
\text { clinical dataset (1 } \\
\text { patient) }\end{array}$ & $\begin{array}{l}\text { Min-max normalization; output } \\
\text { filtering in (2); training data: four-day } \\
\text { protocol (1), testing data: } 3 \text {-day } \\
\text { scenario and in vivo data over a month } \\
\text { (2) }\end{array}$ & $\begin{array}{l}\text { Average RMSE for } \\
\text { PH of } 60 \text { minutes: } \\
11.72(1), 21.09 \text { (2) }\end{array}$ & $\begin{array}{l}\text { Linearized average } \\
\text { model }(46.82(2)) \text {, } \\
\text { daily model } \\
\text { predictor }\end{array}$ \\
\hline 80 & $\begin{array}{l}\text { Prediction } \\
\text { of } \mathrm{HbAlc}{ }^{\dagger}\end{array}$ & $\begin{array}{l}\text { 1-D CNN, } \\
\text { Inception } \\
\text { module, FC } \\
\text { layers }\end{array}$ & $\begin{array}{l}\text { A clinical dataset } \\
\text { (759 T1D subjects, } \\
1543 \text { observations) }\end{array}$ & $\begin{array}{l}\text { Behavioral feature extraction; manual } \\
\text { feature extraction; } 10 \text {-fold } \\
\text { cross-validation; batch normalization; } \\
\text { loss regularization }\end{array}$ & $\begin{array}{l}\text { Mean absolute error: } \\
4.80 \text {, the coefficient } \\
\text { of determination: } \\
0.71\end{array}$ & $\begin{array}{l}\text { Nathan's formula, } \\
\text { CNN (5.98, 0.62), } \\
\text { manual features } \\
\text { extraction network }\end{array}$ \\
\hline 81 & $\begin{array}{l}\text { Prediction } \\
\text { of BG } \\
\text { levels }\end{array}$ & $\begin{array}{l}\text { LSTM and } 2 \\
\text { FC layers }\end{array}$ & OhioT1DM dataset ${ }^{\star}$ & $\begin{array}{l}\text { Scaling glucose values by } 0.01 \text {; the } \\
\text { ratio of training, validation and testing } \\
\text { data: } 60 \%, 20 \% \text { and } 20 \%\end{array}$ & $\begin{array}{l}\text { RMSE for } 30,60-\min \\
\text { PH: } 18.867,31.403\end{array}$ & $\begin{array}{l}\text { Previous work } \\
\text { with machine } \\
\text { learning }\end{array}$ \\
\hline
\end{tabular}


Li et al. transferred the prediction into a classification problem and used 1-D dilated CNNs in their GluNet framework to classify the predictive changes of future BG values [77]. The study tested the model on the two clinical datasets. The use of dilated DNNs to improve the BG prediction is also highlighted in [82]-[84]. Similarly, Zaitcev et al. employed 1-D CNNs with the Inception module to estimate $\mathrm{HbA} 1 \mathrm{c}$ from imperfect time series of CGM [80]. An important consideration for BG prediction is whether the algorithm can be applied in real-time. In this regard, the deep learning models based on CNN and LSTM layers have been validated in smartphone applications to perform real-time BG prediction with short inference time and small memory consumption [74], [77].

Another application of CNNs is to estimate macronutrient content as a daily-life support [72]. With a publicly available dataset of food images (Food-101 dataset) [85], the trained CNN model can predict the food category based on the food images from smartphones, then assist decision support systems and AP systems to compute the required amount of meal bolus insulin. The proposed algorithm was validated in the UVA/Padova T1D simulator with the disturbances of carbohydrate content and incorrect estimation of meal sizes. The UVA/Padova T1D simulator, developed by the University of Virginia (US) and the University of Padova (Italy), is a glucose-insulin dynamics simulator that has been accepted by the Food and Drug Administration for pre-clinical studies [86]. In recent years, many research groups use computer simulation, i.e. in silico setup, to test algorithms in various virtual scenarios, considering the high costs and safety concerns associated to actual clinical trials in humans and animals. Fox et al. used the UVA/Padova T1D simulator to test DRL algorithms to control the delivery of basal insulin, using GRU and 1-D CNN architectures. Recent studies also explored the latest DRL algorithms for other types of hormones in glycemic control, such as glucagon and bolus insulin [87], [88]. Moreover, the simulator is frequently employed in glucose prediction to generate synthetic population datasets that are used for initial validation. Although most of the studies conducted experiments on their proprietary clinical datasets, there is a dataset available to researchers, the OhioT1DM dataset, which was released for the first edition of BG level prediction challenge in 2018 and later updated for the 2020 edition [89]. This dataset contains multi-modal data (CGM, meals, insulin, exercise) corresponding to 12 subjects with T1D over eight weeks.

Furthermore, an unsupervised learning algorithm based on DBNs, and taking ECG signals as input, was employed to detect hypoglycemia in children with T1D [68]. Similarly, in a recent study, ECGs were used to detect nocturnal hypoglycemia in healthy individuals with a CNN-LSTM model [90].

\section{Diagnosis of Complications}

In this category, most research has focused on the analysis of medical imaging to detect and diagnose multiple complications associated to people living with diabetes, as shown in Table III Diabetes-related complications are diverse and regular examinations and clinical visits are time-consuming, expensive, and subjective [106]. For a long-term chronic disorder such as diabetes, its treatment is a heavy burden on the healthcare systems. Therefore, automated systems that are able to screen, detect, predict, and diagnose diabetes-related complications play an important role in population-based surveillance and monitoring.

Diabetic retinopathy (DR) is the leading cause of vision impairments and blindness in the world [107]. DR is often difficult to be detected until vision-threatening events occur. Fortunately, the state-of-art technologies of deep learning have shown great potential to meet this challenge and provide solutions to various DR problems reaching, in some cases, superhuman performance [27]. Following the success in the computer version $(\mathrm{CV})$, a large number of $\mathrm{CNN}$-based models have been adopted to extract the representation from retinal fundus photographs. In the 2015 Kaggle competition on DR screening, all the top results were achieved by $\mathrm{CNNs}$, using a publicly available dataset [108]. Other public datasets with images of DR examinations include the Messidor-2 dataset [109] and the E-Ophtha dataset [110]. In Table [II], nearly all the selected studies used CNNs to detect DR (10/11, 91\%). The exception is the study by ElTanboly et al. which designed a multistage deep fusion classification network with a stack of non-negativity-constrained AEs to detect DR in optical coherence tomography (OCT) images for the patients who have almost normal retina appearances [93]. The AE model achieved high classification accuracy on an experiment with 52 subjects. As for $\mathrm{CNN}$-based studies, most of the approaches are adapted or inspired from two popular architectures in the CV; VGGNet $(4 / 10,40 \%)$ and Inception (5/10, 50\%). In [98], multiple popular CNN configurations were explored on the Kaggle dataset, where VGGNet-s obtained the highest accuracy in the experiments. VGGNet was developed by the University of Oxford (UK), aiming at improving the recognition performance on the ImageNet database with small kernel size and deep networks [39], while Inception employs sparse connections between activation functions in an Inception module to enhance the efficiency of computation on GPUs [40]. Both of the architectures achieved satisfactory performance on DR detection. Abràmoff et al. proposed a VGGNetbased model to detect multiple classes of DR on Messidor-2 dataset [91], achieving a high sensitivity of $96.8 \%$ on referable DR. Then the VGG adapted architecture was validated in two large scale datasets with multi-ethnic populations [95], [100]. Their studies indicated deep learning methods can detect the referable DR with high accuracy but with much less time than human assessors. Gulshan et al. used an Inceptionbased architecture to detect referable DR and achieved the sensitivity of $96.8 \%$ and the specificity of $87.0 \%$. The clinical settings to implement such systems were further investigated, including the feasibility and acceptability of outpatient settings [96] and grader variability [97]. Ruamviboonsuk et al. conducted a nationwide experiment to validate an Inceptionbased model [99]. Compared with human specialists, the deep learning model obtained significantly higher sensitivity and slightly lower specificity. Their achievement is regarded as one of the human level performance milestones in the AI Index 2019 annual report [111]. Meanwhile, Arcadu et al. proposed 
TABLE III: Summary of selected articles from the literature on diagnosis of complications.

\begin{tabular}{|c|c|c|c|c|c|c|}
\hline Ref. & Cases & Models & Data Sources & Development Process & Main Outcomes & Baselines \\
\hline [91] & $\begin{array}{l}\text { Referable } \\
\text { DR } \\
\text { detection }\end{array}$ & $\begin{array}{l}\text { CNN (Inspired } \\
\text { by AlexNet, } \\
\text { VGGNet) }\end{array}$ & $\begin{array}{l}\text { (1) EyeCheck } \\
\text { project, (2) } \\
\text { Messidor-2 dataset }{ }^{\star}\end{array}$ & $\begin{array}{l}\text { Training data: } 10,000 \text { to } 1,250,000 \\
\text { unique samples (1), testing data: } \\
1748 \text { images (2) }\end{array}$ & $\begin{array}{l}\text { Sensitivity: } 96.8 \% \text {, } \\
\text { specificity: } 87.0 \% \text {, } \\
\text { AUC: } 0.980\end{array}$ & $\begin{array}{l}\text { Classical detector } \\
\text { by LR (AUC: } \\
0.955) \text { ) }\end{array}$ \\
\hline [92] & $\begin{array}{l}\text { Referable } \\
\text { DR } \\
\text { detection }\end{array}$ & $\begin{array}{l}\text { CNN } \\
\text { (Inception-v3), } \\
\text { an ensemble of } \\
10 \text { networks }\end{array}$ & $\begin{array}{l}\text { (1) EyePACS-1 } \\
\text { dataset, (2) } \\
\text { Messidor-2 dataset } \text { d }^{\star}\end{array}$ & $\begin{array}{l}\text { Batch normalization; pre-initialization } \\
\text { by the ImageNet data; training and } \\
\text { validation data : } 80 \% \text { and } 20 \% \text { of } \\
128,125 \text { retinal images, testing data: } \\
4497 \text { images (1) and } 1748 \text { images (2) }\end{array}$ & $\begin{array}{l}\text { Sensitivity: } 97.5 \%(1), \\
96.1 \%(2), \text { specificity: } \\
93.9 \%(1), 93.4 \%(2), \\
\text { AUC: } 0.990(1), 0.991 \\
\text { (2) }\end{array}$ & N/A \\
\hline 93 & $\begin{array}{l}\text { DR } \\
\text { detection } \ddagger\end{array}$ & $\begin{array}{l}\text { A stack of } \\
\text { non-negativity- } \\
\text { constrained } \\
\text { AEs }\end{array}$ & $\begin{array}{l}\text { Images from } 52 \\
\text { clinical scans with } \\
12 \text { retinal layers }\end{array}$ & $\begin{array}{l}\text { Feature extraction by cumulative } \\
\text { distribution function; training data: } \\
40 \text { subjects, testing data: } 12 \text { subjects }\end{array}$ & $\begin{array}{l}\text { Sensitivity: } 92 \% \text {, } \\
\text { specificity: } 83 \% \text {, } \\
\text { accuracy: } 100 \%\end{array}$ & $\begin{array}{l}\text { k-star }(89 \%, 89 \% \text {, } \\
89 \%), \text { KNN, RF, } \\
\text { DT }\end{array}$ \\
\hline |94 & $\begin{array}{l}\text { DR } \\
\text { detection }\end{array}$ & $\begin{array}{l}\text { Customized } \\
\text { CNN: (5 } \\
\text { residual blocks } \\
\text { ), DT classifier }\end{array}$ & $\begin{array}{l}\text { (1) EyePACS } \\
\text { dataset, (2) } \\
\text { Messidor-2 } \\
\text { dataset }^{\star},(3) \\
\text { E-Ophtha dataset }^{\star}\end{array}$ & $\begin{array}{l}\text { Feature extraction; training and } \\
\text { validation data: } 75,137 \text { images } \\
\text { (5-fold cross-validation, } 1 \text { ), testing } \\
\text { data: } 1368 \text { images (2) and } 405 \\
\text { images (3) }\end{array}$ & $\begin{array}{l}\text { Sensitivity: } 94 \%(1), \\
93 \%(2), 90 \%(3), \\
\text { specificity: } 98 \%(1), \\
87 \%(2), 94 \%(3), \\
\text { AUC: } 0.97(1), 0.94 \\
(2), 0.95(3)\end{array}$ & $\begin{array}{l}\text { Previous work } \\
\text { with machine } \\
\text { learning on (2) }\end{array}$ \\
\hline 95 & $\begin{array}{l}\text { Referable } \\
\text { DR } \\
\text { detection }\end{array}$ & $\begin{array}{l}\text { CNN (Adapted } \\
\text { VGGNet) }\end{array}$ & $\begin{array}{l}\text { Singapore national } \\
\text { DR screening } \\
\text { program }\end{array}$ & $\begin{array}{l}\text { Each image was analyzed by two } \\
\text { graders and one specialist, training } \\
\text { data: } 76,370 \text { images }(2010-2013 \\
\text { year), testing data: } 71,896 \text { images } \\
\text { (2014-2015 year) }\end{array}$ & $\begin{array}{l}\text { Sensitivity: } 90.5 \% \text {, } \\
\text { specificity: } 91.6 \% \text {, } \\
\text { AUC: } 0.936\end{array}$ & N/A \\
\hline 96 & $\begin{array}{l}\text { Referable } \\
\text { DR } \\
\text { detection }\end{array}$ & $\begin{array}{l}\mathrm{CNN} \\
\text { (Inception-v3) }\end{array}$ & LabelMe dataset & $\begin{array}{l}21 \text { grader validated the accuracy of } \\
\text { the labels; training data: } 58,790 \\
\text { images, cross-validation data: } 8000 \\
\text { images }\end{array}$ & $\begin{array}{l}\text { Sensitivity: } 92.3 \% \text {, } \\
\text { specificity: } 93.7 \%, 96 \% \\
\text { of participants satisfied } \\
\text { with the model }\end{array}$ & $\begin{array}{l}\text { Manual screening } \\
\text { models }\end{array}$ \\
\hline 97] & $\begin{array}{l}\text { Moderate or } \\
\text { worse DR } \\
\text { detection }\end{array}$ & $\begin{array}{l}\mathrm{CNN} \\
\text { (Inception-v4), } \\
\text { an ensemble of } \\
10 \text { networks }\end{array}$ & $\begin{array}{l}\text { EyePACS clinics, } \\
\text { (2) Messidor-2 } \\
\text { dataset }^{\star},(3) \\
\text { EyePACS-2 dataset }\end{array}$ & $\begin{array}{l}\text { Gaussian process bandit algorithm } \\
\text { (hyper-parameter tuning); training } \\
\text { and validation data: } 1,665,151 \text { and } \\
3737 \text { images (1), (2), testing data: } \\
1958 \text { images (3) }\end{array}$ & $\begin{array}{l}\text { Sensitivity: } 97.1 \% \text {, } \\
\text { specificity: } 92.3 \% \text {, } \\
\text { AUC: } 0.986\end{array}$ & $\begin{array}{l}\text { Three retinal } \\
\text { Specialists } \\
\text { (sensitivity: } \\
83.8 \% \text { ) }\end{array}$ \\
\hline 98 & $\begin{array}{l}\mathrm{DR} \\
\text { detection }\end{array}$ & $\begin{array}{l}\text { CNN } \\
\text { (VGGNet-s) }\end{array}$ & Kaggle dataset ${ }^{\star}$ & $\begin{array}{l}\text { Normalization schemes and data } \\
\text { augmentation; non-local means } \\
\text { denoising; 5-fold cross-validation, } \\
\text { training and validation data: } 35,126 \\
\text { images }\end{array}$ & $\begin{array}{l}\text { Sensitivity: } 86.47 \% \text {, } \\
\text { specificity: } 97.43 \% \text {, } \\
\text { AUC: } 0.9786, \\
\text { accuracy: } 95.68 \%\end{array}$ & $\begin{array}{l}\text { AlexNet, ResNet, } \\
\text { VGGNet-16, } \\
\text { VGGNet-19 } \\
\text { (specificity: } \\
96.49 \% \text { ), } \\
\text { GoogleNet, }\end{array}$ \\
\hline [99] & $\begin{array}{l}\text { Referable } \\
\text { DR } \\
\text { detection }\end{array}$ & $\begin{array}{l}\mathrm{CNN} \\
\text { (Inception-v4), } \\
\text { an ensemble of } \\
10 \text { networks }\end{array}$ & $\begin{array}{l}\text { A large-scale } \\
\text { population }(13 \\
\text { health regions, } 7517 \\
\text { patients) }\end{array}$ & $\begin{array}{l}\text { A cascade of thresholds } \\
\text { (hyper-parameter tuning); testing } \\
\text { data: } 25,326 \text { images }\end{array}$ & $\begin{array}{l}\text { Sensitivity: } 96.8 \% \text {, } \\
\text { specificity: } 95.6 \% \text {, } \\
\text { AUC: } 0.987\end{array}$ & $\begin{array}{l}13 \text { human regional } \\
\text { graders } \\
\text { (sensitivity: } 74 \% \text { ) }\end{array}$ \\
\hline$[100$ & $\begin{array}{l}\text { Referable } \\
\text { DR } \\
\text { detection }\end{array}$ & $\begin{array}{l}\text { CNN(Adapted } \\
\text { VGGNet) }\end{array}$ & $\begin{array}{l}\text { A multi-ethnic, } \\
\text { multi-site dataset ( } 5 \\
\text { races, } 18,912 \\
\text { patients) }\end{array}$ & $\begin{array}{l}\text { Training and validation data: } 76,370 \\
\text { and } 8000 \text { images, testing data: } \\
93,293 \text { images }\end{array}$ & $\begin{array}{l}\text { The estimation of DR } \\
\text { prevalence: } 16.1 \% \text {, the } \\
\text { AUC for referable DR: } \\
0.863 \text {, the time taken } \\
\text { to diagnose: } 10.4 \mathrm{~h} \text {, risk } \\
\text { factor: } 0.743\end{array}$ & $\begin{array}{l}10 \text { retinal } \\
\text { specialists and } 7 \\
\text { professional } \\
\text { graders } \\
\text { (prevalence: } 15.9 \%, \\
\text { time: } 1554.8 \mathrm{~h} \text { ) }\end{array}$ \\
\hline [101] & $\begin{array}{l}\text { Estimation } \\
\text { of DR } \\
\text { severity } \\
\text { scale }\end{array}$ & $\begin{array}{l}\text { CNN pillars } \\
\text { (Inception-v3) } \\
\text { and RF }\end{array}$ & $\begin{array}{l}\text { (1) Kaggle dataset }{ }^{\star} \text {, } \\
\text { (2) } 2 \text { large clinical } \\
\text { trials (530 patients) }\end{array}$ & $\begin{array}{l}\text { SHAP for feature selection; transfer } \\
\text { learning }(1) ; 5 \text {-fold cross-validation: } \\
4781 \text { images }(2)\end{array}$ & $\begin{array}{l}\text { AUC at month } 6,12 \text {, } \\
24: 0.68,0.79,0.77\end{array}$ & $\begin{array}{l}\text { Well-trained } \\
\text { reading center } \\
\text { experts }\end{array}$ \\
\hline$[102$ & $\begin{array}{l}\text { Prediction } \\
\text { of mortality } \\
\text { in ICU }\end{array}$ & $\begin{array}{l}\text { 1-D CNN and } \\
2 \text { FC layers }\end{array}$ & MIMIC-III dataset ${ }^{\star}$ & $\begin{array}{l}\text { Feature analysis by importance; } \\
\text { addressing imbalance classes; } \\
\text { training and testing data: } 70 \% \text { and } \\
30 \% \text { from } 9000 \text { subjects }\end{array}$ & AUC: 0.885 & $\begin{array}{l}\text { ANN (0.792), RF, } \\
\text { DT }\end{array}$ \\
\hline [103 & $\begin{array}{l}\text { Prediction } \\
\text { of } \\
\text { myocardial } \\
\text { infarction } \ddagger\end{array}$ & DMLP & $\begin{array}{l}\text { American } \\
\text { commercial health } \\
\text { plan }\end{array}$ & $\begin{array}{l}\text { Descriptive statistics analysis, } \\
\text { confounding factor analysis; } \\
\text { extracting } 199,116 \text { patients }\end{array}$ & $\begin{array}{l}\text { AUC: } 0.767 \text {, with } \\
\text { hazard ratio: } 0.81 \text { and } \\
0.63\end{array}$ & LR (AUC: 0.760) \\
\hline $\mid 104$ & $\begin{array}{l}\text { Classification } \\
\text { of diabetic } \\
\text { foot }\end{array}$ & $\begin{array}{l}\text { Customized } \\
\text { 9-layer CNN }\end{array}$ & $\begin{array}{l}\text { Plantar thermogram } \\
\text { database with } 167 \\
\text { subjects }\end{array}$ & $\begin{array}{l}\text { Data augmentation; patch extraction; } \\
\text { the ratio of training and validation } \\
\text { data: } 70 \% \text { and } 30 \%, 10 \text {-fold } \\
\text { cross-validation }\end{array}$ & $\begin{array}{l}\text { Sensitivity: } 0.9167 \text {, } \\
\text { AUC: } 0.8533\end{array}$ & $\begin{array}{l}\text { SVM, ANN, } \\
\text { AlexNet, } \\
\text { GoogLeNet }\end{array}$ \\
\hline [105 & $\begin{array}{l}\text { Detection of } \\
\text { diabetic } \\
\text { neuropathy }{ }^{\dagger \ddagger}\end{array}$ & $\begin{array}{l}\text { U-Net CNNs } \\
(5 \text { ensembles })\end{array}$ & $\begin{array}{l}\text { (1) BioImLab } \\
\text { dataset }^{\star}, \text { (2) Beijing } \\
\text { dataset, (3) ENA } \\
\text { dataset }\end{array}$ & $\begin{array}{l}\text { Patch extraction; training set: } 1698 \\
\text { images (2), testing set: } 2137 \text { images } \\
(1),(3)\end{array}$ & $\begin{array}{l}\text { Fibre length } 0.933 \text {, } \\
\text { length/segment: } 0.656 \text {, } \\
\text { branch points: } 0.891 \text {, } \\
\text { nail points: } 0.623\end{array}$ & $\begin{array}{l}\text { ACCMetrics } \\
\text { model }(0.825 \\
0.325,0.570 \\
0.257)\end{array}$ \\
\hline
\end{tabular}


an Inception model to predict DR progression by leveraging individual color fundus photographs [101].

The deep learning applications to other complications are also noted in the literature. Wittler et al. designed a CNNbased model to predict mortality based on the data from the intensive care unit (ICU) patients, achieving an AUC score of 0.885 [102]. The ICU dataset in this study, referred to as MIMIC-III, is freely accessible [112]. Williams et al. [105] proposed a U-Net CNN to quantify the nerve fiber properties in the diagnosis of diabetic neuropathy, involving a public dataset [113]. Their results show an excellent localization performance for the quantification and the potential to be adopted in clinical settings. In [104], a customized CNN was designed to detect plantar ulcers on the thermography of diabetic foot with a publicly accessible dataset [114]. Moreover, Yamada et al. investigated the incidence of cardiovascular disease among three anti-diabetic drugs, using a DMLP model that achieved better results than conventional LR analysis [103]. In summary, it is noted that most of the studies focused on microvascular complications, including DR [91][101], diabetic foot [104], and diabetic neuropathy [105], while there is only one study focusing on macrovascular complications (cardiovascular diseases) [103].

\section{Summary of Deep Learning Techniques}

A significant number of deep learning methods have been adopted by the diabetes research community, covering various architectures in supervised learning and unsupervised learning. Among these, CNN-based architectures are the most widely used, particularly in clinical imaging problems. CNNs are good at extracting features from raw data, requiring little hand-engineered work and domain expertise on image processing [23]. Hence, the main application of CNNs has been in the analysis of clinical scans and medical images used for the diagnosis of diabetes-related complications. Another use of CNN-based architectures is to support the daily life of people with diabetes by estimating macronutrients from food images. Today the new techniques are actively developed in the $\mathrm{CV}$ area to improve the model performance while reducing the complexity. Hence the evolution of CNN configurations (VGGNet, Inception) opens the door to the development of more powerful algorithms. In addition, some studies have applied 1-D CNNs to process sequential signals, using convolutional filters to extract data features with a large receptive field. Several of these work further used LSTM layers to process the outcomes from $\mathrm{CNN}$ architectures to compute the temporal dependencies by hybrid models: convolutional RNN (CRNN) [74] and CNN-LSTM [54], [90], relying on the powerful capability of RNNs in sequence processing. In fact, RNN-based architectures dominate among the glucose management applications, especially for BG prediction. The recursive computations and advanced cell structures are suitable for mapping the glucose series measured by CGM in real-time. Some studies explored the latest advances of the techniques in NLP, such as bidirectional LSTM [70] and neural attention mechanisms [51], [76].

DMLP and unsupervised learning algorithms are commonly used in diabetes diagnosis. However, careful feature selection and normalization in pre-processing are required in many cases of these tasks, due to the heterogeneous forms of the records in EHR datasets. To this end, conventional machine learning algorithms are employed to discover the most relevant features. For instance, in [59], PCA was employed to calculate principal components scores for each data feature by deriving the eigenvectors coefficients and weights. In [60], LR analysis was used to compute correlations between non-invasive variables and the attributes of individuals to select significant features for diabetes detection.

Aiming at specific tasks, the DNN layers can be accessed and customized to incorporate with other models. Apart from the CRNN and the CNN-LSTM, other hybrid learning models in the literature involve the linear model [57], SVM [54], DTs [94], and RFs [101] to integrate data features at the input or perform a second-level analysis at the output. Moreover, the ensemble models for deep learning are highlighted in [92], [97], [99], [101], [105]. In these works, the ensemble contains multiple CNNs trained by the same dataset and obtains the final results by linear averaging in the testing phase. Due to the random initialization and batch feeding, each of the CNNs learns a distinct representation and improves the overall accuracy and generalization.

Training a very deep model from scratch is time-consuming since millions of parameters in the DNN units need to be tuned. In this regard, an approach called transfer learning, i.e. pre-training, provides a shortcut to solve this issue. Particularly, for clinical imaging tasks, the ImageNet database is a critical auxiliary component for CNN pre-training, which has been used in [72], [92]. Fine-tuning the weights based on the ImageNet paradigms can largely speed up convergence for target datasets, but it is not a necessary step if there are sufficient computational resources [115]. In the tasks of glucose management, the in silico datasets derived from the simulators and a portion of real clinical data are used for pretraining [69], [70], [76]. It is an effective method to mitigate the high demand for data during the DNN training. The unsupervised pre-training in DBNs is calculated layer by layer [47] to find good initial weights for the discriminative fine-tuning, which is used by [59]. Alternatively, data augmentation is a way to improve model performance with limited data, which can be found in [98], [104]. These studies used a series of image manipulation, such as shifting, rotating, and flipping, to transform the available images and expand the training sets.

\section{DISCUSSION}

\section{A. Limitations and Challenges}

Although deep learning has improved the state of art in several areas of diabetes, the applications in healthcare systems need to be robust, reliable, and convincing to avoid safety issues and provide effective therapeutic aids. In this context, there remain several limitations and challenges for deep learning to be further introduced in actual clinical settings. Table IV summarizes the five common limitations identified from the selected articles: data volume, data variability, data quality, feature processing, and interpretability. In real-world scenarios, the data collected from people with diabetes are 
prone to be imperfect, due to human errors and sensor artifacts. The process to collect real data is sometimes expensive and time-consuming. Due to data privacy policies, sharing datasets among research teams is sometimes difficult. These factors lead to many studies employing a reduced, sometimes insufficient, amount of data. Another challenge that arises due to the complexity of glucose dynamics is how to process the available data in order to characterize people with diabetes. Also, deep learning models lack transparency. From the perspective of clinicians, why the models produce the output for a certain input case is important, particularly for some critical decision-making applications. The complicated structures in DNN layers can effectively learn the patterns from non-linear signals but reduce the interpretability of the model. Therefore, it is crucial to consider the trade-off between performance and interpretability when investigating deep learning for diabetes. Finally, the efficiency of training deep learning models is expected to be enhanced through new algorithmic and hardware developments [52], [104].

\section{B. Opportunities and Future Work}

The list of challenges introduced in Section $\mathrm{V}-\mathrm{A}$ not only applies to the field of diabetes but also is valid in other health domains. Deep learning is a hotspot in the era of AI, and it is worth noting that most of the selected papers are publications from the recent two years, as shown in Fig. 4, which indicates that this is an emerging technology. Hence, there is a large space to improve the current applications for diabetes.

First, the digital records and vital signs are increasingly collected by the multi-modal systems with wearables and smartphone applications. Most of these data are conveniently uploaded to centralized systems or cloud repositories. With the popularization of the Internet of things and 5G networks, data volumes and variability of data sources are expected to significantly increase in many healthcare applications, and in particular, in diabetes care. As the data volume expands, many low-quality data samples can be filtered out and removed from training sets, and the advances in wearables (e.g. CGM) can effectively reduce the measurement errors. Deep learning is well adapted to cope with such an increase in data availability. Several publicly available datasets are outlined in Section IV and more datasets will be shared in the communities after proper post-processing and de-anonymization. In order to deploy deep learning in an ambulatory setting, the frameworks mentioned in Section II can be easily ported to mobile devices by using tools such as TensorFlow Lite [74], [77].

To interpret deep learning technologies in healthcare, many recent attempts in the AI domain have been made to enhance model transparency and understand model functionality. In particular, a unified framework, the SHapley Additive exPlanations (SHAP), was proposed to explain the input features that contribute to the final output, which has been validated on many data-driven applications in healthcare domains [116]. This is also an effective method to select input features by ranking their importance. In Table III], an article also employed SHAP analysis to attribute the descriptors for the CNN outcomes [101]. Another effective technique to interpret the learned features of CNN layers is t-distributed stochastic neighbor embedding (t-SNE) [117], which was used to visualize the clusters of heartbeat data according to glucose levels in [90]. The use of t-SNE can also be generalized to other CNN applications, such as DR detection, to qualitatively analyze the extracted feature maps. Moreover, a recent study also verified the conformance of neural network models in terms of glucose-insulin dynamics [118]. Similar approaches can be used to analyze the performance of DNNs and further enhance interpretability.

Instead of solely using data-driven models, integrating the expert knowledge in the learning process can help to better understand the underlying mechanisms of a health condition such as diabetes. Specifically, there are two feasible methods. One is to incorporate the physiological parameters as the input feature of the models, and the other is to use expert knowledge as a guide during the training process. Expert knowledge is also essential to craft safety constrains and calculate the confidence of the model outputs.

Many selected articles mentioned that their studies require to be further validated in real-world scenarios [79], [80], [95], [101], [105]. In this regard, a team from Google took a step forward. They conducted a human-centered study in 11 clinics, applying deep learning to diabetic eye diseases [119]. The results indicate that some socio-environmental factors need to be addressed before the widespread deployment of such automated systems.

\section{CONCLUSION}

In this paper, we present a comprehensive review of the current trend in deep learning technologies for diabetes research. We performed a systematic search, selected a collection of articles, and summarized the key information focusing on three areas: diagnosis of diabetes, glucose management, and diagnosis of diabetes related complications. In these areas, various DNN architectures and learning techniques have been applied and obtained superior experimental performance that previous conventional machine learning approached. On the other hand, several challenges have been identified from the literature including data availability, feature processing, and model interpretability. In the future, there is great potential to meet these challenges by transferring the latest advances in deep learning technologies into massive multi-modal data of diabetes management. We expect that deep learning technologies will be widespread in clinical settings and largely improve the treatment of people living with diabetes.

\section{ACKNOWLEDGMENT}

The work has been supported by EPSRC EP/P00993X/1 and the President's PhD Scholarship at Imperial College London.

\section{REFERENCES}

[1] P. Saeedi, I. Petersohn, P. Salpea, B. Malanda, S. Karuranga, N. Unwin, S. Colagiuri, L. Guariguata, A. A. Motala, K. Ogurtsova et al., "Global and regional diabetes prevalence estimates for 2019 and projections for 2030 and 2045: Results from the International Diabetes Federation Diabetes Atlas," Diabetes research and clinical practice, vol. 157, p. 107843, 2019. 
TABLE IV: Summary of the limitations and challenges identified by the selected articles.

\begin{tabular}{|c|c|c|}
\hline Category & Description & References \\
\hline Data volume & $\begin{array}{l}\text { Training a deep model for complicated tasks requires a high volume of data. Collecting data from people } \\
\text { with diabetes is often time-consuming and expensive, compared to other tasks in CV and NLP. Consequently, } \\
\text { many studies face a shortage of data during their research cycles. }\end{array}$ & $\begin{array}{l}[51], \mid 53], \mid 54], \mid 56], \\
{[73],[81],[91],} \\
[98],[101], \mid 104]\end{array}$ \\
\hline Data variability & $\begin{array}{l}\text { The variability among people with diabetes is large due to the complex glucose dynamics. To obtain better } \\
\text { generalization for deep learning models, the training data needs to cover a diverse range of individuals, such } \\
\text { as people of different ages and comorbidities. However, many datasets are often collected from a specific } \\
\text { cohort of people, which lacks diversity and could bring bias to the learning. }\end{array}$ & 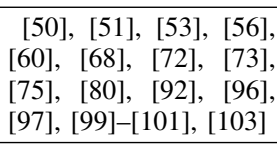 \\
\hline Data quality & $\begin{array}{l}\text { Similar to many other problems in healthcare, most of the diabetes datasets are heterogeneous, sparse, and } \\
\text { noisy with some missing values. It is not realistic to collect perfect data from either clinical practice or daily } \\
\text { self-management, e.g. the unavoidable errors from CGM sensors. }\end{array}$ & 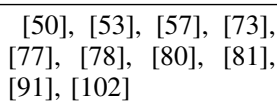 \\
\hline $\begin{array}{l}\text { Feature } \\
\text { processing }\end{array}$ & $\begin{array}{l}\text { The major challenge in feature processing is to find the most effective features for models to learn the } \\
\text { representations. Manually screening and analyzing each feature in a diabetes dataset could require a lot of } \\
\text { engineering work, but using automated data-driven methods, such as PCA, would ignore some physiological } \\
\text { knowledge and rely too much on the characteristics of the data. A more comprehensive analysis of additional } \\
\text { factors and features is needed with the advances in data collecting and physiological models. }\end{array}$ & 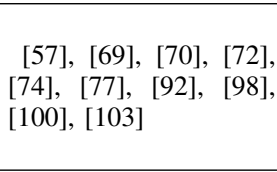 \\
\hline Interpretability & $\begin{array}{l}\text { The interpretability, i.e. explainability, stands for how the model obtains the corresponding output based on } \\
\text { a set of inputs. It is an important goal for AI applications in healthcare to convince clinicians to adopt } \\
\text { such systems. In many cases, deep learning models are regarded as "black boxes" with a lack of model } \\
\text { transparency due to complex nonlinear layers. As a consequence, if the model performance degrades in } \\
\text { certain circumstances, it might be difficult to explain why. }\end{array}$ & 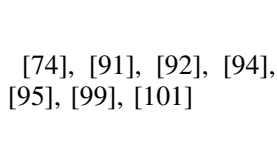 \\
\hline
\end{tabular}

[2] World Health Organization, Global report on diabetes. World Health Organization, 2016

[3] American Diabetes Association and others, "2. classification and diagnosis of diabetes," Diabetes care, vol. 40, no. Supplement 1, pp. S11-S24, 2017

[4] Centers for Disease Control and Prevention and others, "National diabetes fact sheet: national estimates and general information on diabetes and prediabetes in the united states, 2011," Atlanta, GA: US department of health and human services, centers for disease control and prevention, vol. 201, no. 1, pp. 2568-2569, 2011.

[5] T. Tuomi, N. Santoro, S. Caprio, M. Cai, J. Weng, and L. Groop, "The many faces of diabetes: a disease with increasing heterogeneity," The Lancet, vol. 383, no. 9922, pp. 1084-1094, 2014.

[6] J. C. Pickup, "Management of diabetes mellitus: is the pump mightier than the pen?" Nature Reviews Endocrinology, vol. 8, no. 7, p. 425, 2012.

[7] A. D. Deshpande, M. Harris-Hayes, and M. Schootman, "Epidemiology of diabetes and diabetes-related complications," Physical therapy, vol. 88 , no. 11 , pp. 1254-1264, 2008.

[8] R. D. Coffen and L. M. Dahlquist, "Magnitude of type 1 diabetes self-management in youth health care needs diabetes educators," The Diabetes Educator, vol. 35, no. 2, pp. 302-308, 2009.

[9] J. Vora and T. Heise, "Variability of glucose-lowering effect as a limiting factor in optimizing basal insulin therapy: a review," Diabetes, Obesity and Metabolism, vol. 15, no. 8, pp. 701-712, 2013.

[10] D. C. Klonoff, "Continuous glucose monitoring: roadmap for 21st century diabetes therapy," Diabetes care, vol. 28, no. 5, pp. 1231-1239, 2005.

[11] Juvenile Diabetes Research Foundation Continuous Glucose Monitoring Study Group, "Continuous glucose monitoring and intensive treatment of type 1 diabetes," New England Journal of Medicine, vol. 359, no. 14, pp. 1464-1476, 2008.

[12] D. Rodbard, "Continuous glucose monitoring: a review of successes, challenges, and opportunities," Diabetes technology \& therapeutics, vol. 18, no. S2, pp. S2-3, 2016.

[13] P. Herrero, M. El-Sharkawy, J. Daniels, N. Jugnee, C. N. Uduku, M. Reddy, N. Oliver, and P. Georgiou, "The bio-inspired artificial pancreas for type 1 diabetes control in the home: System architecture and preliminary results," Journal of diabetes science and technology, vol. 13, no. 6, pp. 1017-1025, 2019.

[14] C. Fabris and B. Kovatchev, "The closed-loop artificial pancreas in 2020," Artificial Organs, 2020.

[15] A. Slomski, "Artificial pancreas improves glycemic control," Jama, vol. 320, no. 20, pp. 2068-2068, 2018.

[16] T. S. Bailey and J. Y. Stone, "A novel pen-based bluetooth-enabled insulin delivery system with insulin dose tracking and advice," Expert opinion on drug delivery, vol. 14, no. 5, pp. 697-703, 2017.

[17] M. Grady, L. B. Katz, H. Cameron, and B. L. Levy, "Diabetes apprelated text messages from health care professionals in conjunction with a new wireless glucose meter with a color range indicator improves glycemic control in patients with type 1 and type 2 diabetes:
Randomized controlled trial," JMIR diabetes, vol. 2, no. 2, p. e19, 2017.

[18] L. F. Garabedian, D. Ross-Degnan, and J. F. Wharam, "Mobile phone and smartphone technologies for diabetes care and self-management," Current diabetes reports, vol. 15, no. 12, p. 109, 2015.

[19] F. L. Schwartz, C. R. Marling, and R. C. Bunescu, "The promise and perils of wearable physiological sensors for diabetes management," Journal of diabetes science and technology, vol. 12, no. 3, pp. 587-591, 2018.

[20] P. B. Jensen, L. J. Jensen, and S. Brunak, "Mining electronic health records: towards better research applications and clinical care," Nature Reviews Genetics, vol. 13, no. 6, p. 395, 2012.

[21] C. M. Bishop, Pattern recognition and machine learning. springer, 2006.

[22] D. Ravì, C. Wong, F. Deligianni, M. Berthelot, J. Andreu-Perez, B. Lo, and G.-Z. Yang, "Deep learning for health informatics," IEEE journal of biomedical and health informatics, vol. 21, no. 1, pp. 4-21, 2016.

[23] Y. LeCun, Y. Bengio, and G. Hinton, "Deep learning," Nature, vol 521, no. 7553, pp. 436-444, 2015.

[24] M. Rigla, G. García-Sáez, B. Pons, and M. E. Hernando, "Artificial intelligence methodologies and their application to diabetes," Journal of diabetes science and technology, vol. 12, no. 2, pp. 303-310, 2018.

[25] I. Contreras and J. Vehi, "Artificial intelligence for diabetes management and decision support: literature review," Journal of medical Internet research, vol. 20, no. 5, p. e10775, 2018.

[26] I. Dankwa-Mullan, M. Rivo, M. Sepulveda, Y. Park, J. Snowdon, and $\mathrm{K}$. Rhee, "Transforming diabetes care through artificial intelligence: the future is here," Population health management, vol. 22, no. 3, pp 229-242, 2019.

[27] A. Grzybowski, P. Brona, G. Lim, P. Ruamviboonsuk, G. S. Tan, M. Abramoff, and D. S. Ting, "Artificial intelligence for diabetic retinopathy screening: a review," Eye, pp. 1-10, 2019.

[28] I. Goodfellow, Y. Bengio, and A. Courville, Deep learning. MIT press, 2016.

[29] D. E. Rumelhart, G. E. Hinton, and R. J. Williams, "Learning representations by back-propagating errors," Nature, vol. 323, no. 6088, pp. 533-536, 1986.

[30] A. Krizhevsky, I. Sutskever, and G. E. Hinton, "ImageNet classification with deep convolutional neural networks," in Advances in neural information processing systems, 2012, pp. 1097-1105.

[31] J. Bergstra, O. Breuleux, F. Bastien, P. Lamblin, R. Pascanu, G. Desjardins, J. Turian, D. Warde-Farley, and Y. Bengio, "Theano: a CPU and GPU math expression compiler," in Proceedings of the Python for scientific computing conference (SciPy), vol. 4, no. 3. Austin, TX, 2010.

[32] Y. Jia, E. Shelhamer, J. Donahue, S. Karayev, J. Long, R. Girshick, S. Guadarrama, and T. Darrell, "Caffe: Convolutional architecture for fast feature embedding," in Proceedings of the 22nd ACM international conference on Multimedia, 2014, pp. 675-678.

[33] M. Abadi, P. Barham, J. Chen, Z. Chen, A. Davis, J. Dean, M. Devin, S. Ghemawat, G. Irving, M. Isard et al., "Tensorflow: A system for large-scale machine learning," in 12th $\{$ USENIX $\}$ Symposium on 
Operating Systems Design and Implementation ( $\{O S D I\}$ 16), 2016, pp. 265-283.

[34] F. Seide and A. Agarwal, "CNTK: Microsoft's open-source deeplearning toolkit," in Proceedings of the 22nd ACM SIGKDD International Conference on Knowledge Discovery and Data Mining, 2016, pp. 2135-2135

[35] A. Paszke, S. Gross, F. Massa, A. Lerer, J. Bradbury, G. Chanan, T. Killeen, Z. Lin, N. Gimelshein, L. Antiga et al., "Pytorch: An imperative style, high-performance deep learning library," in Advances in Neural Information Processing Systems, 2019, pp. 8024-8035.

[36] Y. LeCun, L. Bottou, Y. Bengio, and P. Haffner, "Gradient-based learning applied to document recognition," Proceedings of the IEEE vol. 86 , no. 11 , pp. $2278-2324,1998$.

[37] D. C. Cireşan, U. Meier, L. M. Gambardella, and J. Schmidhuber, "Deep, big, simple neural nets for handwritten digit recognition," Neural computation, vol. 22, no. 12, pp. 3207-3220, 2010.

[38] J. Deng, W. Dong, R. Socher, L.-J. Li, K. Li, and L. Fei-Fei, "ImageNet: A large-scale hierarchical image database," in 2009 IEEE conference on computer vision and pattern recognition. IEEE, 2009, pp. 248-255.

[39] K. Simonyan and A. Zisserman, "Very deep convolutional networks for large-scale image recognition," in 3rd International Conference on Learning Representations, ICLR, Y. Bengio and Y. LeCun, Eds., 2015.

[40] C. Szegedy, W. Liu, Y. Jia, P. Sermanet, S. Reed, D. Anguelov, D. Erhan, V. Vanhoucke, and A. Rabinovich, "Going deeper with convolutions," in Proceedings of the IEEE conference on computer vision and pattern recognition, 2015, pp. 1-9.

[41] K. He, X. Zhang, S. Ren, and J. Sun, "Deep residual learning for image recognition," in Proceedings of the IEEE conference on computer vision and pattern recognition, 2016, pp. 770-778.

[42] Y. Bengio, P. Simard, and P. Frasconi, "Learning long-term dependencies with gradient descent is difficult," IEEE transactions on neural networks, vol. 5, no. 2, pp. 157-166, 1994

[43] S. Hochreiter and J. Schmidhuber, "Long short-term memory," Neural computation, vol. 9, no. 8, pp. 1735-1780, 1997.

[44] K. Cho, B. van Merriënboer, D. Bahdanau, and Y. Bengio, "On the properties of neural machine translation: Encoder-decoder approaches," in Proceedings of SSST-8, Eighth Workshop on Syntax, Semantics and Structure in Statistical Translation. Association for Computational Linguistics, 2014, pp. 103-111.

[45] A. Vaswani, N. Shazeer, N. Parmar, J. Uszkoreit, L. Jones, A. N. Gomez, Ł. Kaiser, and I. Polosukhin, "Attention is all you need," in Advances in neural information processing systems, 2017, pp. 59986008.

[46] G. E. Hinton, "Deep belief networks," Scholarpedia, vol. 4, no. 5, p. $5947,2009$.

[47] G. E. Hinton, S. Osindero, and Y.-W. Teh, "A fast learning algorithm for deep belief nets," Neural computation, vol. 18, no. 7, pp. 15271554, 2006.

[48] V. Mnih, K. Kavukcuoglu, D. Silver, A. A. Rusu, J. Veness, M. G. Bellemare, A. Graves, M. Riedmiller, A. K. Fidjeland, G. Ostrovski et al., "Human-level control through deep reinforcement learning," Nature, vol. 518, no. 7540, p. 529, 2015.

[49] D. Moher, A. Liberati, J. Tetzlaff, and D. G. Altman, "Preferred reporting items for systematic reviews and meta-analyses: the PRISMA statement," Annals of internal medicine, vol. 151, no. 4, pp. 264-269, 2009.

[50] R. Miotto, L. Li, B. A. Kidd, and J. T. Dudley, "Deep patient: an unsupervised representation to predict the future of patients from the electronic health records," Scientific reports, vol. 6, no. 1, pp. 1-10, 2016

51] T. Pham, T. Tran, D. Phung, and S. Venkatesh, "Predicting healthcare trajectories from medical records: A deep learning approach," Journal of biomedical informatics, vol. 69, pp. 218-229, 2017.

52] S. Ramesh, H. Balaji, N. C. S. Iyengar, and R. D. Caytiles, "Optima predictive analytics of Pima diabetics using deep learning," International Journal of Database Theory and Application, vol. 10, no. 9, pp 47-62, 2017

[53] S. Lekha and M. Suchetha, "Real-time non-invasive detection and classification of diabetes using modified convolution neural network," IEEE journal of biomedical and health informatics, vol. 22, no. 5, pp. 1630-1636, 2017.

[54] G. Swapna, R. Vinayakumar, and K. Soman, "Diabetes detection using deep learning algorithms," ICT Express, vol. 4, no. 4, pp. 243-246, 2018 .

[55] A. Ashiquzzaman, A. K. Tushar, M. R. Islam, D. Shon, K. Im, J.H. Park, D.-S. Lim, and J. Kim, "Reduction of overfitting in diabetes prediction using deep learning neural network," in IT Convergence and Security 2017. Springer, 2018, pp. 35-43.
[56] S. Spänig, A. Emberger-Klein, J.-P. Sowa, A. Canbay, K. Menrad, and D. Heider, "The virtual doctor: An interactive clinical-decision-support system based on deep learning for non-invasive prediction of diabetes," Artificial intelligence in medicine, vol. 100, p. 101706, 2019.

[57] B. P. Nguyen, H. N. Pham, H. Tran, N. Nghiem, Q. H. Nguyen, T. T. Do, C. T. Tran, and C. R. Simpson, "Predicting the onset of type 2 diabetes using wide and deep learning with electronic health records," Computer methods and programs in biomedicine, vol. 182, p. 105055 , 2019.

[58] K. Kannadasan, D. R. Edla, and V. Kuppili, "Type 2 diabetes data classification using stacked autoencoders in deep neural networks," Clinical Epidemiology and Global Health, vol. 7, no. 4, pp. 530-535, 2019.

[59] P. Prabhu and S. Selvabharathi, "Deep belief neural network model for prediction of diabetes mellitus," in 2019 3rd International Conference on Imaging, Signal Processing and Communication (ICISPC). IEEE, 2019, pp. 138-142.

[60] K. S. Ryu, S. W. Lee, E. Batbaatar, J. W. Lee, K. S. Choi, and H. S. Cha, "A deep learning model for estimation of patients with undiagnosed diabetes," Applied Sciences, vol. 10, no. 1, p. 421, 2020.

[61] N. Cho, J. Shaw, S. Karuranga, Y. Huang, J. da Rocha Fernandes, A. Ohlrogge, and B. Malanda, "IDF Diabetes Atlas: Global estimates of diabetes prevalence for 2017 and projections for 2045," Diabetes research and clinical practice, vol. 138, pp. 271-281, 2018

[62] American Diabetes Association and others, "Screening for diabetes," Diabetes care, vol. 25, no. suppl 1, pp. s21-s24, 2002.

[63] D. Dua and C. Graff, "UCI machine learning repository," 2017. [Online]. Available: http://archive.ics.uci.edu/ml

[64] R. Rossi and N. Ahmed, "The network data repository with interactive graph analytics and visualization," in Twenty-Ninth AAAI Conference on Artificial Intelligence, 2015.

[65] R. S. Lindsay, T. Funahashi, R. L. Hanson, Y. Matsuzawa, S. Tanaka, P. A. Tataranni, W. C. Knowler, and J. Krakoff, "Adiponectin and development of type 2 diabetes in the Pima Indian population," The Lancet, vol. 360, no. 9326, pp. 57-58, 2002.

[66] Icahn School of Medicine at Mount Sinai, "Mount Sinai Data Warehouse," 2015. [Online]. Available: https://labs.icahn.mssm.edu/ msdw/

[67] Practice Fusion, Inc, "Practice Fusion." [Online]. Available: https: //www.practicefusion.com/

[68] P. P. San, S. H. Ling, and H. T. Nguyen, "Deep learning framework for detection of hypoglycemic episodes in children with type 1 diabetes," in 2016 38th Annual International Conference of the IEEE Engineering in Medicine and Biology Society (EMBC). IEEE, 2016, pp. 3503-3506.

[69] S. Mirshekarian, R. Bunescu, C. Marling, and F. Schwartz, "Using LSTMs to learn physiological models of blood glucose behavior," in 2017 39th Annual International Conference of the IEEE Engineering in Medicine and Biology Society (EMBC). IEEE, 2017, pp. 2887-2891.

[70] Q. Sun, M. V. Jankovic, L. Bally, and S. G. Mougiakakou, "Predicting blood glucose with an LSTM and Bi-LSTM based deep neural network," in 2018 14th Symposium on Neural Networks and Applications (NEUREL). IEEE, 2018, pp. 1-5.

[71] I. Fox, L. Ang, M. Jaiswal, R. Pop-Busui, and J. Wiens, "Deep multi-output forecasting: Learning to accurately predict blood glucose trajectories," in Proceedings of the 24th ACM SIGKDD International Conference on Knowledge Discovery \& Data Mining, 2018, pp. 13871395.

[72] A. Chakrabarty, F. J. Doyle, and E. Dassau, "Deep learning assisted macronutrient estimation for feedforward-feedback control in artificial pancreas systems," in 2018 Annual American Control Conference (ACC). IEEE, 2018, pp. 3564-3570.

[73] S. H. A. Faruqui, Y. Du, R. Meka, A. Alaeddini, C. Li, S. Shirinkam, and J. Wang, "Development of a deep learning model for dynamic forecasting of blood glucose level for type 2 diabetes mellitus: Secondary analysis of a randomized controlled trial," JMIR mHealth and uHealth, vol. 7, no. 11, p. e14452, 2019.

[74] K. Li, J. Daniels, C. Liu, P. Herrero-Vinas, and P. Georgiou, "Convolutional recurrent neural networks for glucose prediction," IEEE journal of biomedical and health informatics, 2019.

[75] A. Aliberti, I. Pupillo, S. Terna, E. Macii, S. Di Cataldo, E. Patti, and A. Acquaviva, "A multi-patient data-driven approach to blood glucose prediction," IEEE Access, vol. 7, pp. 69311-69325, 2019.

[76] S. Mirshekarian, H. Shen, R. Bunescu, and C. Marling, "LSTMs and neural attention models for blood glucose prediction: Comparative experiments on real and synthetic data," in 201941 st Annual International Conference of the IEEE Engineering in Medicine and Biology Society $(E M B C)$. IEEE, 2019, pp. 706-712.

[77] K. Li, C. Liu, T. Zhu, P. Herrero, and P. Georgiou, "Glunet: A deep learning framework for accurate glucose forecasting," IEEE journal of biomedical and health informatics, 2019 
[78] I. Fox and J. Wiens, "Reinforcement learning for blood glucose control: Challenges and opportunities," in Reinforcement Learning for Real Life (RLARealLife) Workshop in 36th International Conference on Machine Learning (ICML), 2019.

[79] E. M. Aiello, G. Lisanti, L. Magni, M. Musci, and C. Toffanin, "Therapy-driven deep glucose forecasting," Engineering Applications of Artificial Intelligence, vol. 87, p. 103255, 2020.

[80] A. Zaitcev, M. R. Eissa, Z. Hui, T. Good, J. Elliott, and M. Benaissa, "A deep neural network application for improved prediction of HbA1c in type 1 diabetes," IEEE Journal of Biomedical and Health Informatics, 2020

[81] J. Martinsson, A. Schliep, B. Eliasson, and O. Mogren, "Blood glucose prediction with variance estimation using recurrent neural networks," Journal of Healthcare Informatics Research, vol. 4, no. 1, pp. 1-18, 2020.

[82] T. Zhu, K. Li, P. Herrero, J. Chen, and P. Georgiou, "A deep learning algorithm for personalized blood glucose prediction." in The 3rd International Workshop on Knowledge Discovery in Healthcare Data, IJCAI-ECAI 2018, 2018, pp. 64-78.

[83] J. Chen, K. Li, P. Herrero, T. Zhu, and P. Georgiou, "Dilated recurrent neural network for short-time prediction of glucose concentration." in The 3rd International Workshop on Knowledge Discovery in Healthcare Data, IJCAI-ECAI 2018, 2018, pp. 69-73.

[84] T. Zhu, K. Li, P. Herrero, J. Chen, and P. Georgiou, "Dilated recurrent neural networks for glucose forecasting in type 1 diabetes," Journal of Healthcare Informatics Research, pp. 1-17, 2020.

[85] L. Bossard, M. Guillaumin, and L. Van Gool, "Food-101-mining discriminative components with random forests," in European conference on computer vision. Springer, 2014, pp. 446-461.

[86] C. Dalla Man, F. Micheletto, D. Lv, M. Breton, B. Kovatchev, and C. Cobelli, "The UVA/PADOVA type 1 diabetes simulator: new features," Journal of diabetes science and technology, vol. 8, no. 1, pp. 26-34, 2014.

[87] T. Zhu, K. Li, P. Herrero, and P. Georgiou, "Basal glucose control in type 1 diabetes using deep reinforcement learning: An in silico validation," IEEE Journal of Biomedical and Health Informatics, pp. $1-1,2020$.

[88] T. Zhu, K. Li, L. Kuang, P. Herrero, and P. Georgiou, "An insulin bolus advisor for type 1 diabetes using deep reinforcement learning,' Sensors, vol. 20, no. 18, p. 5058, 2020.

[89] C. Marling and R. Bunescu, "The OhioT1DM dataset for blood glucose level prediction: Update 2020," in The 5th KDH workshop, ECAI 2020 2020, pp. 71-74.

[90] M. Porumb, S. Stranges, A. Pescapè, and L. Pecchia, "Precision medicine and artificial intelligence: A pilot study on deep learning for hypoglycemic events detection based on ECG," Scientific Reports, vol. 10, no. 1 , pp. 1-16, 2020 .

[91] M. D. Abràmoff, Y. Lou, A. Erginay, W. Clarida, R. Amelon, J. C. Folk, and M. Niemeijer, "Improved automated detection of diabetic retinopathy on a publicly available dataset through integration of deep learning," Investigative ophthalmology \& visual science, vol. 57, no. 13, pp. 5200-5206, 2016

[92] V. Gulshan, L. Peng, M. Coram, M. C. Stumpe, D. Wu, A. Narayanaswamy, S. Venugopalan, K. Widner, T. Madams, J. Cuadros et al., "Development and validation of a deep learning algorithm for detection of diabetic retinopathy in retinal fundus photographs," Jama, vol. 316, no. 22, pp. 2402-2410, 2016.

[93] A. ElTanboly, M. Ismail, A. Shalaby, A. Switala, A. El-Baz, S. Schaal, G. Gimel'farb, and M. El-Azab, "A computer-aided diagnostic system for detecting diabetic retinopathy in optical coherence tomography images," Medical physics, vol. 44, no. 3, pp. 914-923, 2017.

[94] R. Gargeya and T. Leng, "Automated identification of diabetic retinopathy using deep learning," Ophthalmology, vol. 124, no. 7, pp. 962-969, 2017.

[95] D. S. W. Ting, C. Y.-L. Cheung, G. Lim, G. S. W. Tan, N. D. Quang, A. Gan, H. Hamzah, R. Garcia-Franco, I. Y. San Yeo, S. Y. Lee et al., "Development and validation of a deep learning system for diabetic retinopathy and related eye diseases using retinal images from multiethnic populations with diabetes," Jama, vol. 318, no. 22, pp. 2211-2223, 2017

[96] S. Keel, P. Y. Lee, J. Scheetz, Z. Li, M. A. Kotowicz, R. J. MacIsaac, and M. He, "Feasibility and patient acceptability of a novel artificial intelligence-based screening model for diabetic retinopathy at endocrinology outpatient services: a pilot study," Scientific reports, vol. 8, no. 1, pp. 1-6, 2018.

[97] J. Krause, V. Gulshan, E. Rahimy, P. Karth, K. Widner, G. S. Corrado, L. Peng, and D. R. Webster, "Grader variability and the importance of reference standards for evaluating machine learning models for diabetic retinopathy," Ophthalmology, vol. 125, no. 8, pp. 1264-1272, 2018.
[98] S. Wan, Y. Liang, and Y. Zhang, "Deep convolutional neural networks for diabetic retinopathy detection by image classification," Computer \& Electrical Engineering, vol. 72, pp. 274-282, 2018.

[99] P. Ruamviboonsuk, J. Krause, P. Chotcomwongse, R. Sayres, R. Raman, K. Widner, B. J. Campana, S. Phene, K. Hemarat, M. Tadarati et al., "Deep learning versus human graders for classifying diabetic retinopathy severity in a nationwide screening program," NPJ digital medicine, vol. 2, no. 1, pp. 1-9, 2019.

[100] D. S. Ting, C. Y. Cheung, Q. Nguyen, C. Sabanayagam, G. Lim, Z. W. Lim, G. S. Tan, Y. Q. Soh, L. Schmetterer, Y. X. Wang et al., "Deep learning in estimating prevalence and systemic risk factors for diabetic retinopathy: A multi-ethnic study," NPJ digital medicine, vol. 2, no. 1 , pp. 1-8, 2019.

[101] F. Arcadu, F. Benmansour, A. Maunz, J. Willis, Z. Haskova, and M. Prunotto, "Deep learning algorithm predicts diabetic retinopathy progression in individual patients," NPJ digital medicine, vol. 2, no. 1 , pp. 1-9, 2019.

[102] I. Wittler, X. Liu, and A. Dong, "Deep learning enabled predicting modeling of mortality of diabetes mellitus patients," in Proceedings of the Practice and Experience in Advanced Research Computing on Rise of the Machines (learning), 2019, pp. 1-6.

[103] T. Yamada, K. Iwasaki, S. Maedera, K. Ito, T. Takeshima, H. Noma, and N. Shojima, "Myocardial infarction in type 2 diabetes using sodiumglucose cotransporter-2 inhibitors, dipeptidyl peptidase-4 inhibitors, or glucagon-like peptide-1 receptor agonists: Proportional hazards analysis by deep neural network-based machine learning," Current Medical Research and Opinion, no. just-accepted, pp. 1-1, 2019.

[104] I. Cruz-Vega, D. Hernandez-Contreras, H. Peregrina-Barreto, J. d. J. Rangel-Magdaleno, and J. M. Ramirez-Cortes, "Deep learning classification for diabetic foot thermograms," Sensors, vol. 20, no. 6, p. 1762, 2020.

[105] B. M. Williams, D. Borroni, R. Liu, Y. Zhao, J. Zhang, J. Lim, B. Ma V. Romano, H. Qi, M. Ferdousi et al., "An artificial intelligence-based deep learning algorithm for the diagnosis of diabetic neuropathy using corneal confocal microscopy: A development and validation study," Diabetologia, vol. 63, no. 2, pp. 419-430, 2020.

[106] E. W. Gregg, N. Sattar, and M. K. Ali, "The changing face of diabetes complications," The lancet Diabetes \& endocrinology, vol. 4, no. 6, pp. $537-547,2016$

[107] J. W. Yau, S. L. Rogers, R. Kawasaki, E. L. Lamoureux, J. W. Kowalski, T. Bek, S.-J. Chen, J. M. Dekker, A. Fletcher, J. Grauslund et al., "Global prevalence and major risk factors of diabetic retinopathy," Diabetes care, vol. 35, no. 3, pp. 556-564, 2012.

[108] Kaggle, Inc, "Diabetic retinopathy detection," 2015. [Online] Available: https://www.kaggle.com/c/diabetic-retinopathy-detection

[109] LaTIM Laboratory and the Messidor program partners, "Messidor-2." [Online]. Available: http://www.adcis.net/en/third-party/messidor2/

[110] PANR-TECSAN-TELEOPHTA, "E-ophtha." [Online]. Available: http: //www.adcis.net/en/third-party/e-ophtha/

[111] R. Perrault, Y. Shoham, E. Brynjoltsson, J. Clark, J. Etchemendy, B. Grosz, T. Lyons, J. Manyika, and S. Niebles, "The AI Index 2019 annual report." 2019.

[112] A. E. Johnson, T. J. Pollard, L. Shen, H. L. Li-wei, M. Feng, M. Ghassemi, B. Moody, P. Szolovits, L. A. Celi, and R. G. Mark, "MIMIC-III, a freely accessible critical care database," Scientific data, vol. 3, p. $160035,2016$.

[113] University of Padova, "BioImLab." [Online]. Available: http://bioimlab. dei.unipd.it/

[114] D. H.-C. H. P.-B. J. R.-M. F. Renero-Carrillo, "Plantar thermogram database for the study of diabetic foot complications," 2019. [Online]. Available: http://dx.doi.org/10.21227/tm4t-9n15

[115] K. He, R. Girshick, and P. Dollár, "Rethinking ImageNet pre-training," in Proceedings of the IEEE International Conference on Computer Vision, 2019, pp. 4918-4927.

[116] S. M. Lundberg, B. Nair, M. S. Vavilala, M. Horibe, M. J. Eisses, T. Adams, D. E Liston, D. K.-W. Low, S.-F. Newman, J. Kim et al., "Explainable machine-learning predictions for the prevention of hypoxaemia during surgery," Nature biomedical engineering, vol. 2, no. 10, pp. 749-760, 2018.

[117] L. v. d. Maaten and G. Hinton, "Visualizing data using t-SNE," Journal of machine learning research, vol. 9, no. Nov, pp. 2579-2605, 2008.

[118] T. Kushner, S. Sankaranarayanan, and M. Breton, "Conformance verification for neural network models of glucose-insulin dynamics," in Proceedings of the 23rd International Conference on Hybrid Systems: Computation and Control, 2020, pp. 1-12.

[119] E. Beede, E. Baylor, F. Hersch, A. Iurchenko, L. Wilcox, P. Ruamviboonsuk, and L. M. Vardoulakis, "A human-centered evaluation of deep learning system deployed in clinics for the detection of diabetic retinopathy," in Proceedings of the 2020 CHI Conference on Human Factors in Computing Systems, 2020, pp. 1-12. 